Supporting Information

\title{
Antibiofouling Ultrathin Poly(amidoxime) Membrane for Enhanced U(VI) Recovery from Wastewater and Seawater
}

Ye Sun,,$^{\dagger}$ Rongrong Liu, ${ }^{\dagger}$ Shunxi Wen,,$^{\dagger}$ Jiawen Wang,,$^{\dagger}$ Lin Chen, ${ }^{\dagger}$ Bingjie Yan, ${ }^{\dagger}$ Shuyi Peng,,$^{\dagger}$ Chao

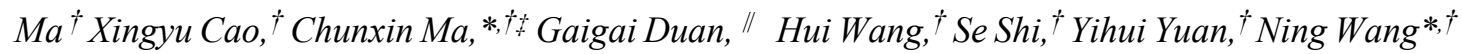

†State Key Laboratory of Marine Resource Utilization in South China Sea, Hainan University, Haikou 570228, P. R. China

\#esearch Institute of Zhejiang University-Taizhou, Taizhou 318000, P. R. China

" International Innovation Center for Forest Chemicals and Materials, College of Materials Science and Engineering, Nanjing Forestry University, Nanjing, 210037, China.

KEYWORDS: uranium recovery, amidoxime, antibiofouling, cellulose nanocrystals, ultrathin membrane

E-mail: machunxin@hainanu.edu.cn,wangn02@foxmail.com 


\section{Experimental Section}

Calculation of the Uranium Adsorption Amount:

According to the literature, ${ }^{1}$ Arsenazo (III) can cooperate with uranyl ions and has a strong specific peak at $652 \mathrm{~nm}$. Therefore, U-concentration can be determined by UV-Vis absorption spectrum of Arsenazo (III). Based on the principle above, we obtained two concentration-absorbance curves that can be used to determine U-concentration in U-added ultrapure water (Figure S1) and uranium-spiked natural seawater (Figure S2) respectively: A standard solution composed of $3.0 \mathrm{~mL}$ ultrapure water, $1.0 \mathrm{~mL}$ of $500 \mathrm{ppm}$ arsenazo (III) aqueous solution, $0.5 \mathrm{~mL}$ of $0.1 \mathrm{~mol} / \mathrm{L} \mathrm{HCl}$ and $0.5 \mathrm{~mL}$ of uranium-added solution. The uranium content of the standard solution showed a gradient change by adjusting the concentration of uranium solution. The concentration absorbance regression curve can be fitted by detecting the absorbance change of standard solution with a UV spectrophotometer. The uranium solution with unknown concentration can be prepared into a mixture according to the above method, and then its concentration can be calculated by using the obtained curvilinear regression.

An AUPM with a dry weight of $10 \mathrm{mg}$ was immersed into a $1.0 \mathrm{~L}$ U-spiked ultrapurewater or U-spiked seawater solutions and stirred with a table concentrator, the uranium adsorption mass of the adsorbent can be calculated based on the formula (1): 


$$
M_{\mathrm{u}}=\left(C_{\mathrm{o}}-C_{\mathrm{t}}\right) \times V
$$

Where $\mathrm{M}_{\mathrm{u}}$ is the uranium adsorption mass of the membrane, $\mathrm{C}_{\mathrm{o}}$ and $\mathrm{C}_{\mathrm{t}}$ are the uranium concentration of the U-spiked aqueous solution at different time respectively, $\mathrm{V}$ is the volume of the U-spiked solution.

Method of the Uranium Desorption Performance:

According to the reported literature, ${ }^{2} 11.7 \mathrm{ml} 30 \% \mathrm{H}_{2} \mathrm{O}_{2}$ aqueous solution and $106 \mathrm{~g}$ $\mathrm{Na}_{2} \mathrm{CO}_{3}$ were dissolved ultrapure water to prepare the $1000 \mathrm{~mL}$ elution solution. The U-uptake AUPM with a dry weight of $10 \mathrm{mg}$ was immersed in the eluent (200 mL). After stirring for $10 \mathrm{~min}$, the adsorbed uranium in the membrane can be desorbed. We can regenerate the membrane through immersing it in $500 \mathrm{~mL}$ pure water and changing the water several times until the $\mathrm{pH}=7$. The regenerated AUPM was utilized for the next adsorption-desorption cycle. 


\section{Data analysis}

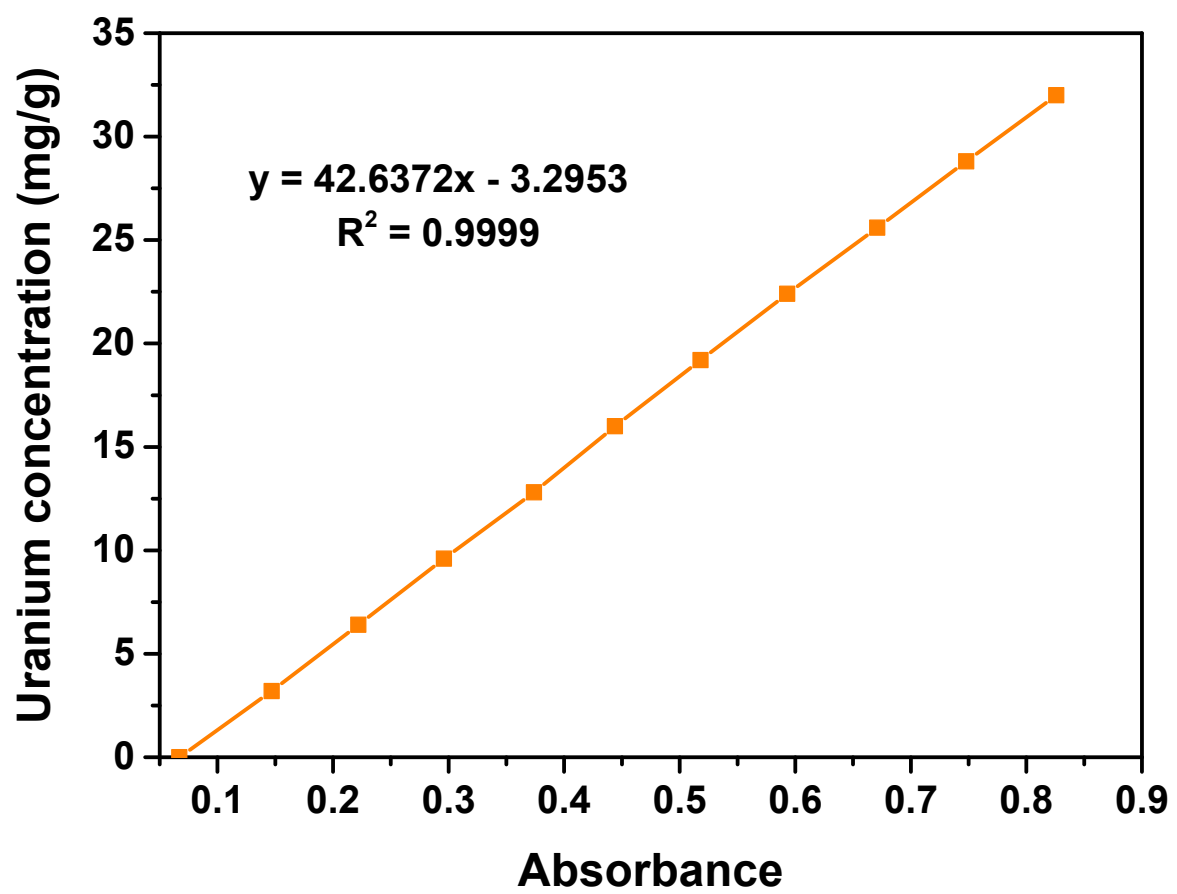

Figure S1. The curvilinear regression of uranium concentration-absorbance in uranium-spiked ultrapure water. 


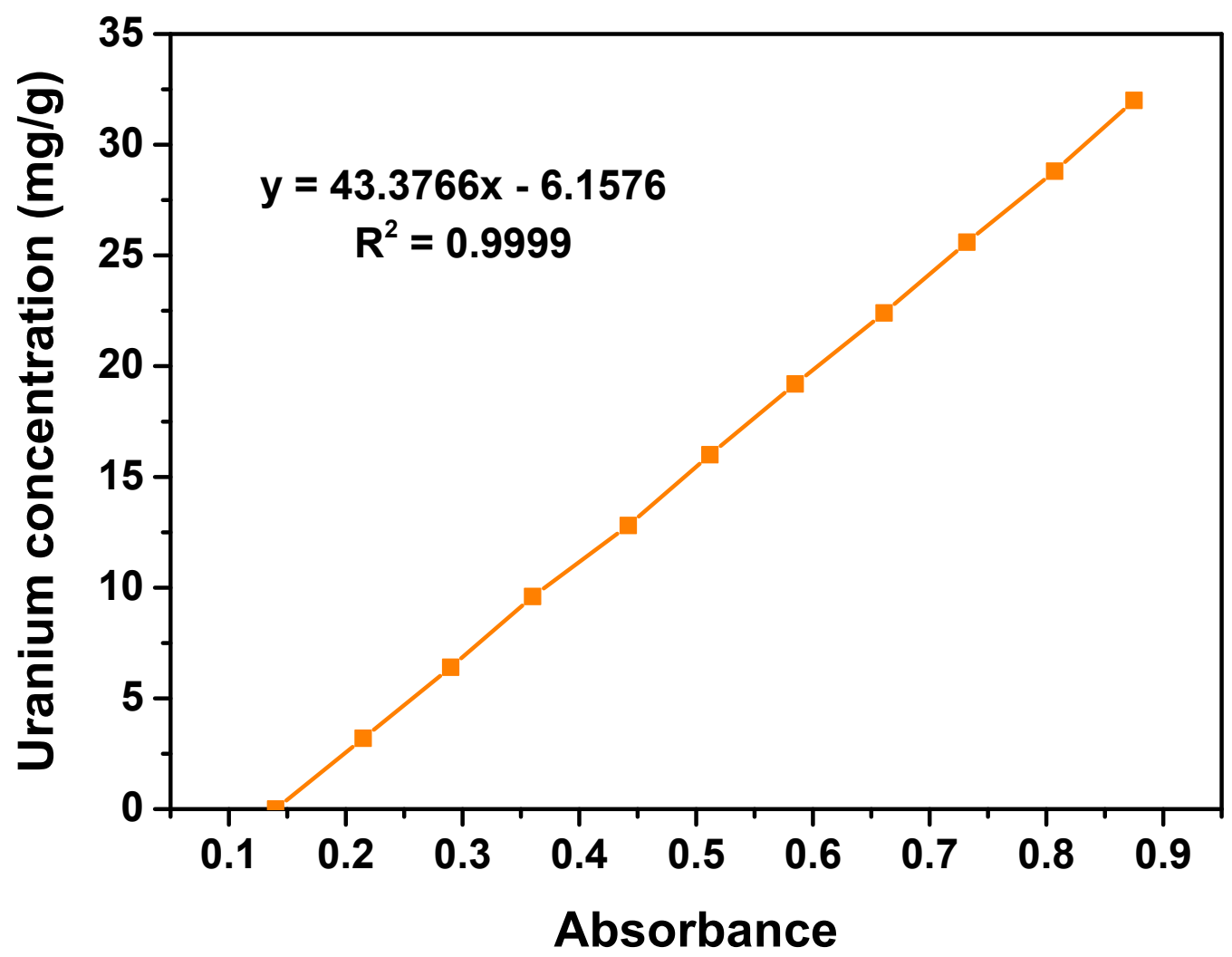

Figure S2. The curvilinear regression of uranium concentration-absorbance in uranium-spiked natural seawater. 
(a)

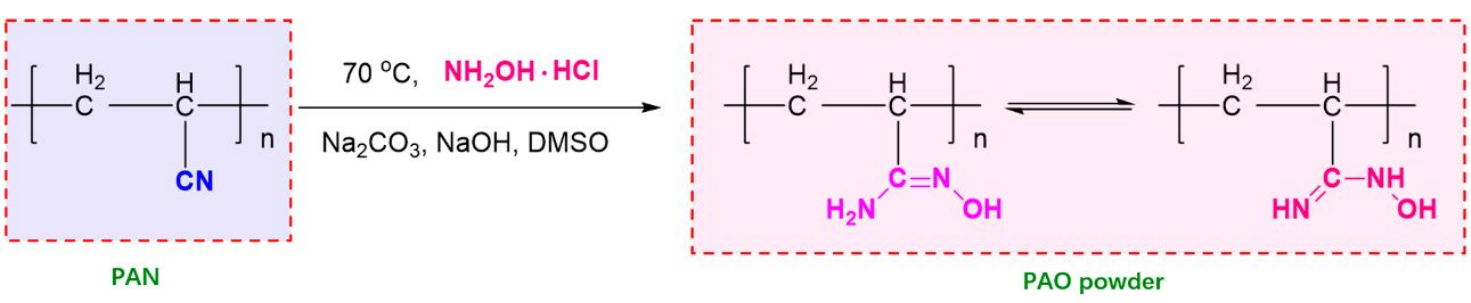

(b)

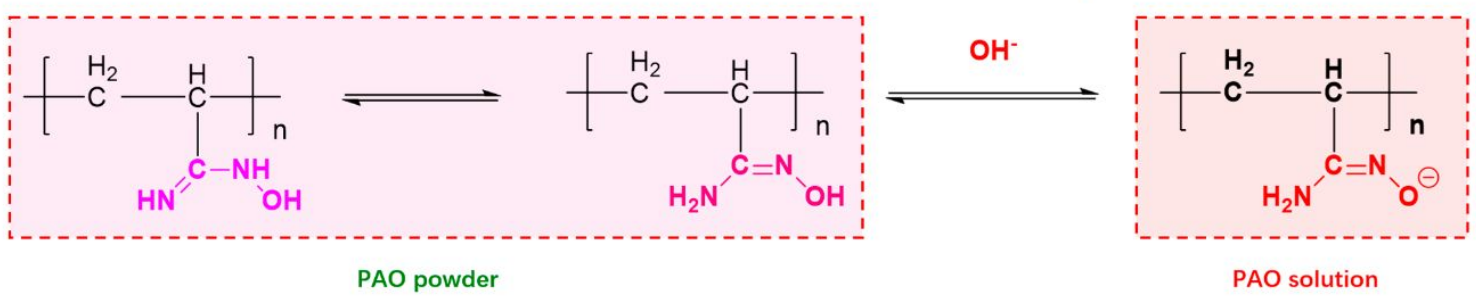

Figure S3. (a) Synthesis of the PAO; (b) Transformation from PAO powder to the PAO alkaline solution. 


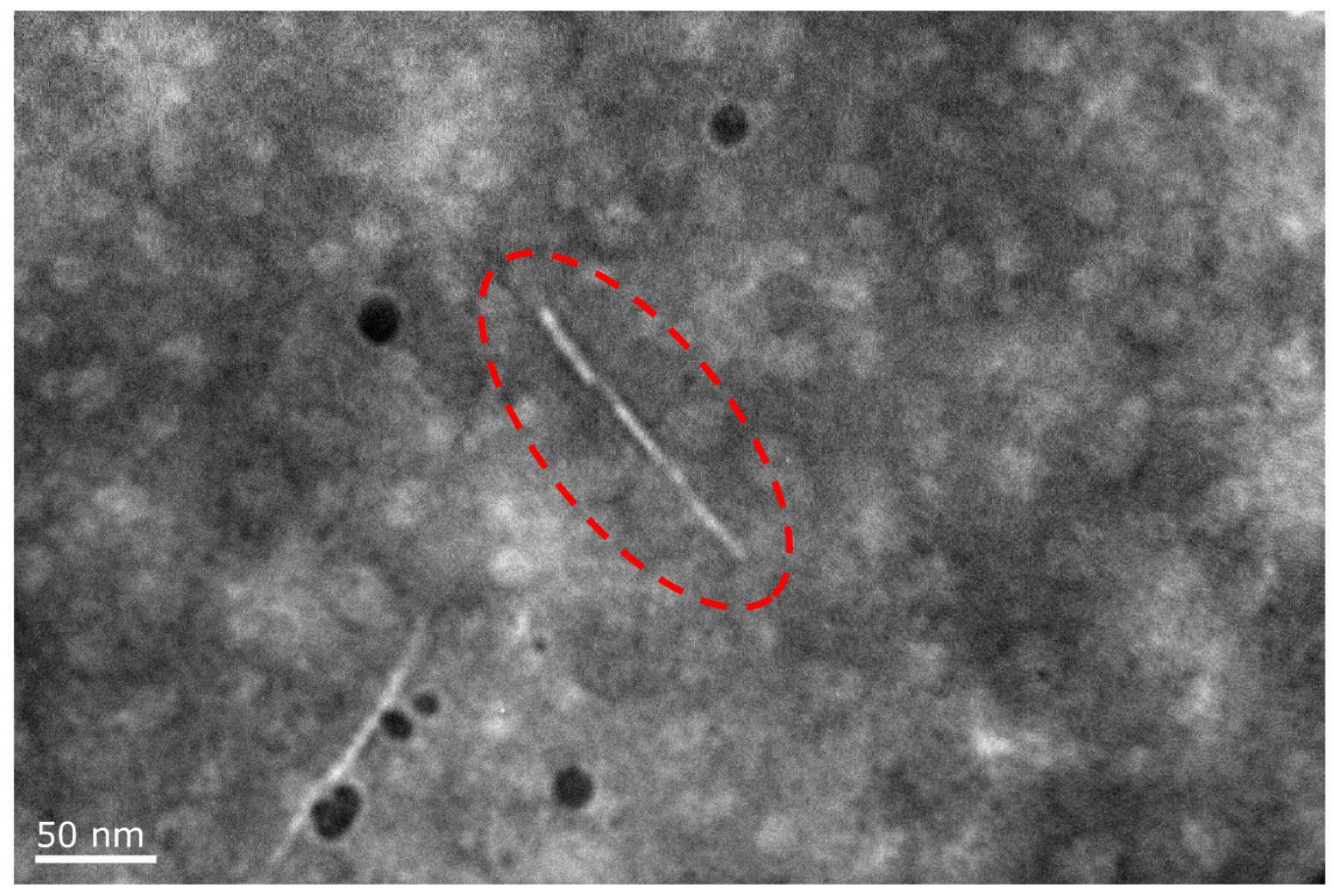

Figure S4. The TEM images of S-CNC. 

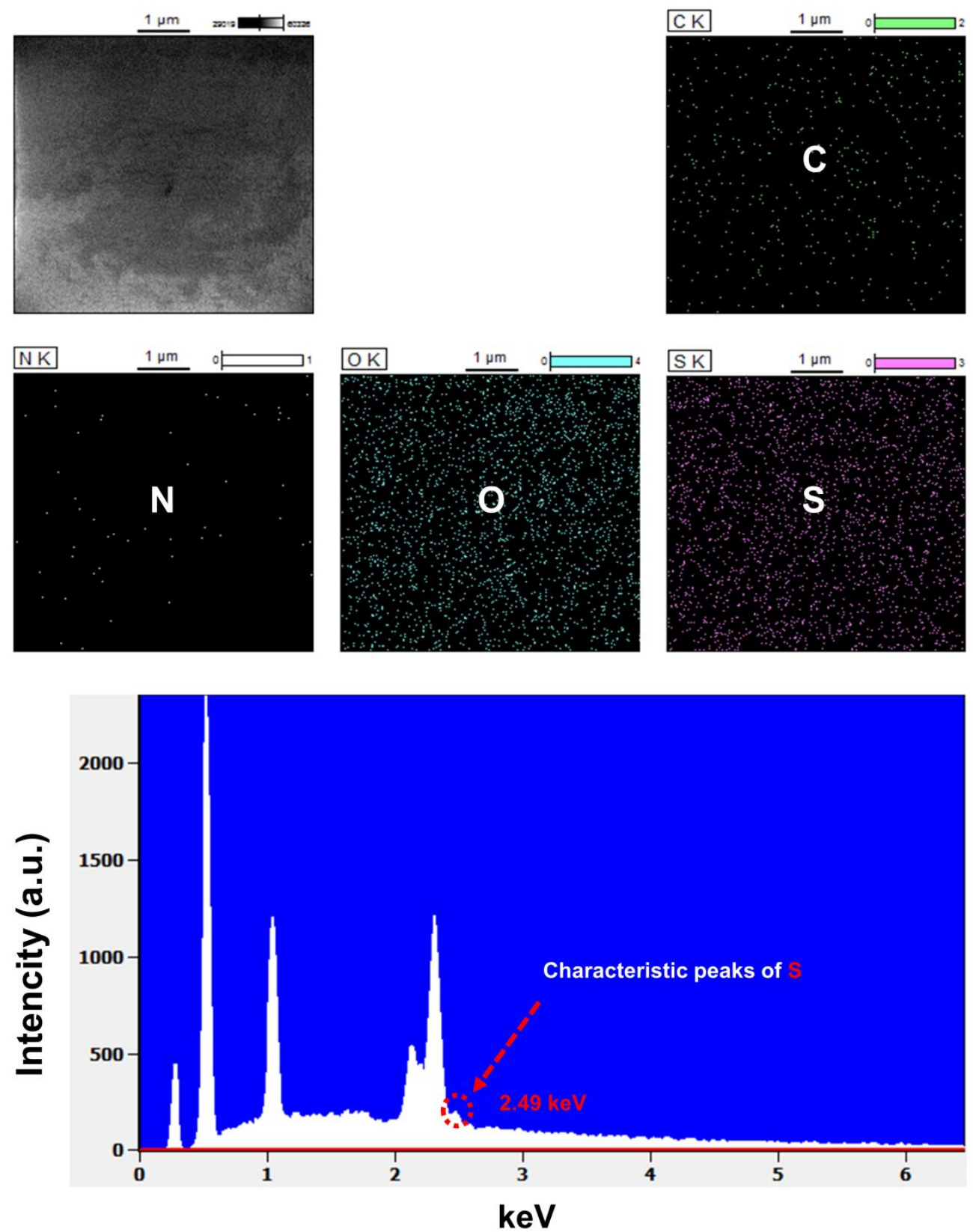

Figure S5. The C, N, O and S EDS mappings of the S-CNC. 


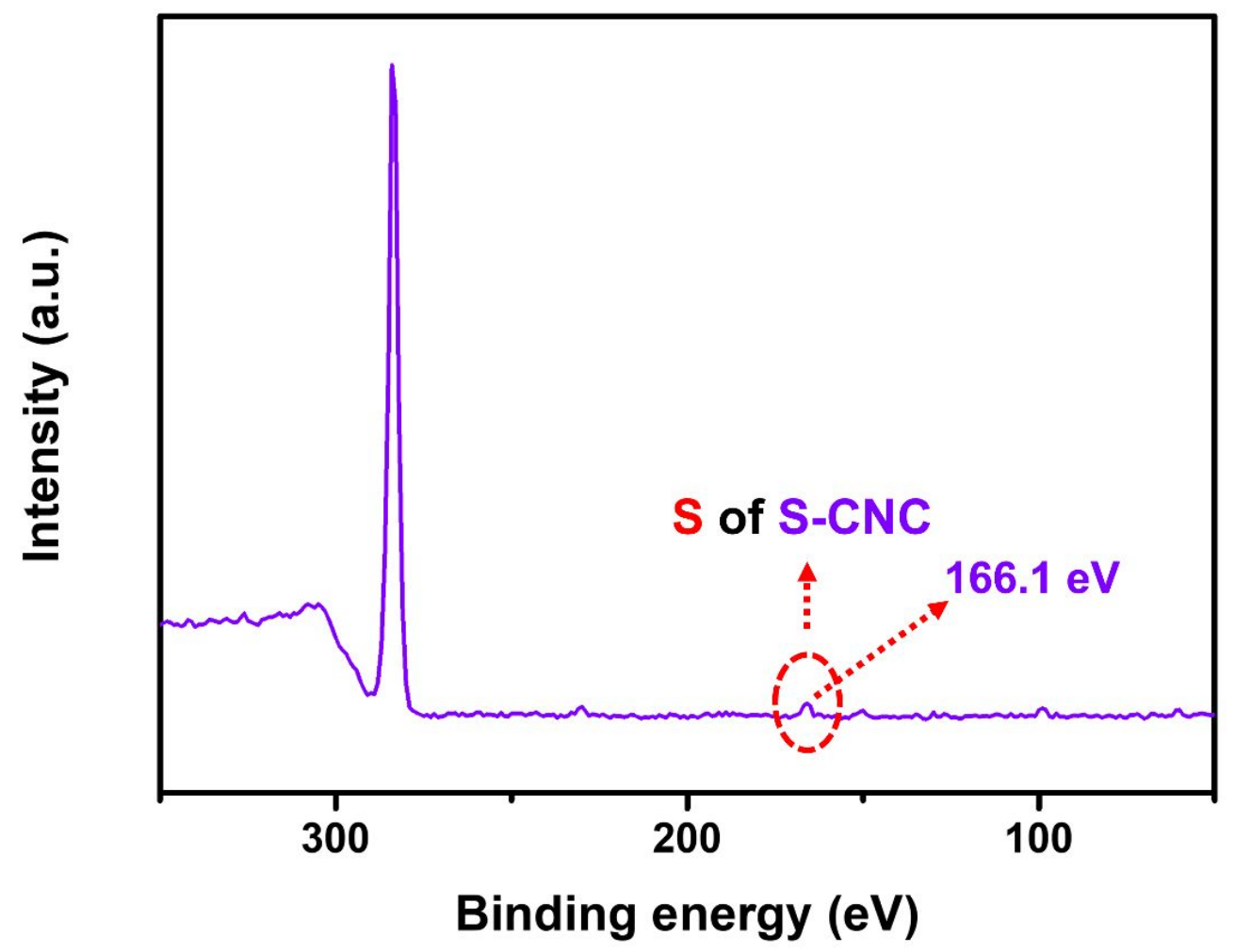

Figure S6. The survey XPS spectra of S-CNC. 

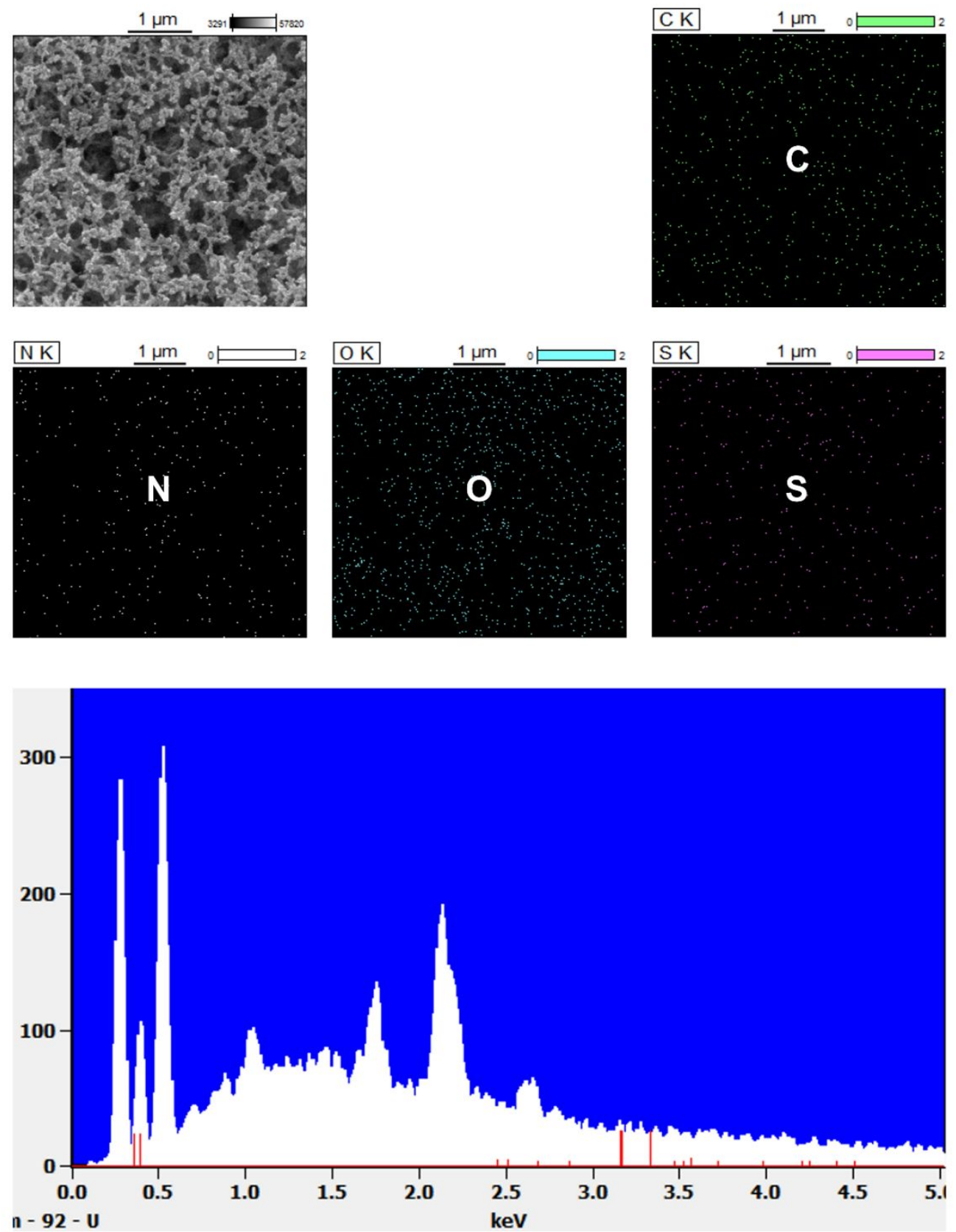

Figure S7. The C, N, O and S EDS mappings of the non-S-CNC blank membrane. 

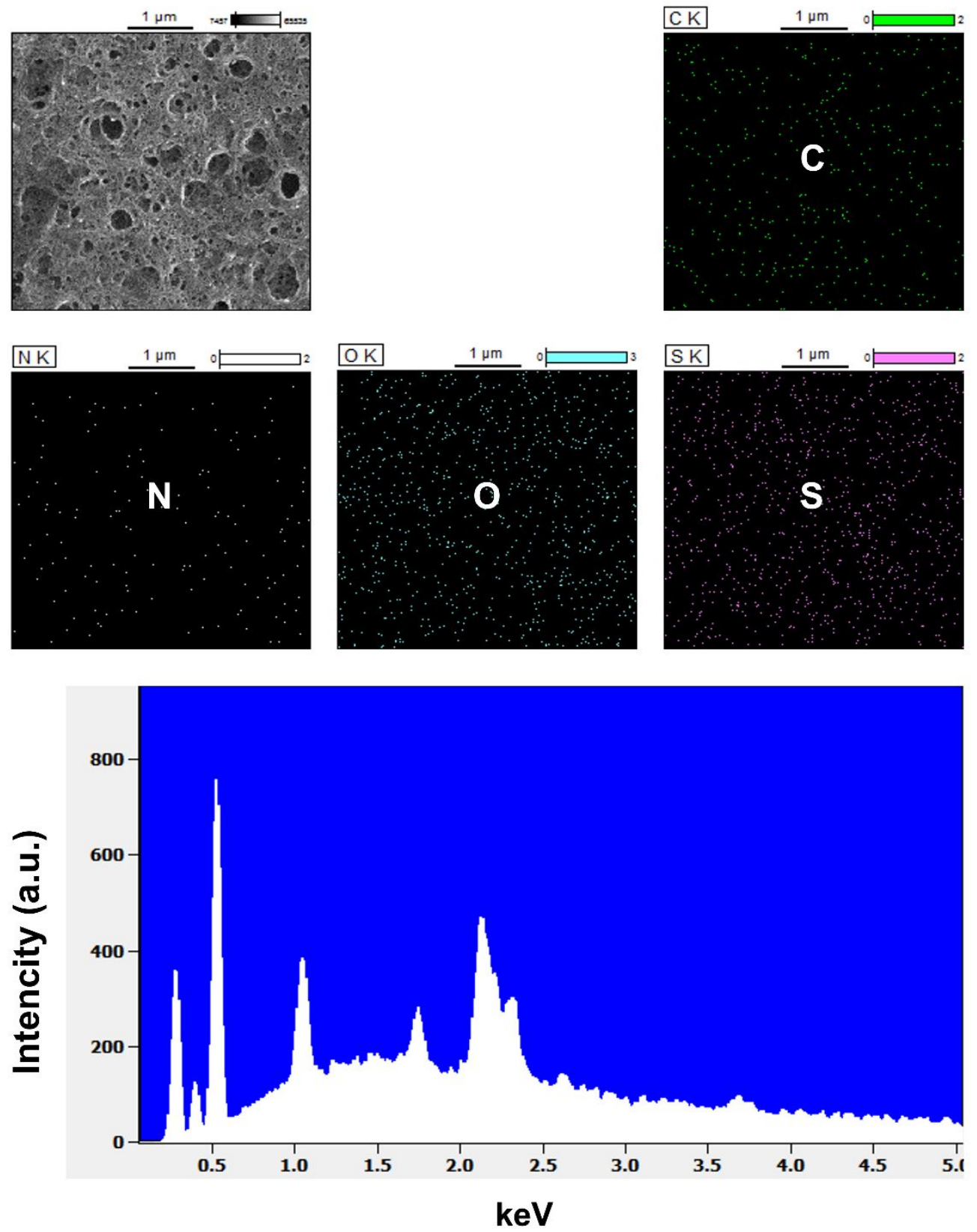

Figure S8. The C, N, O and S EDS mappings of the AUPM. 


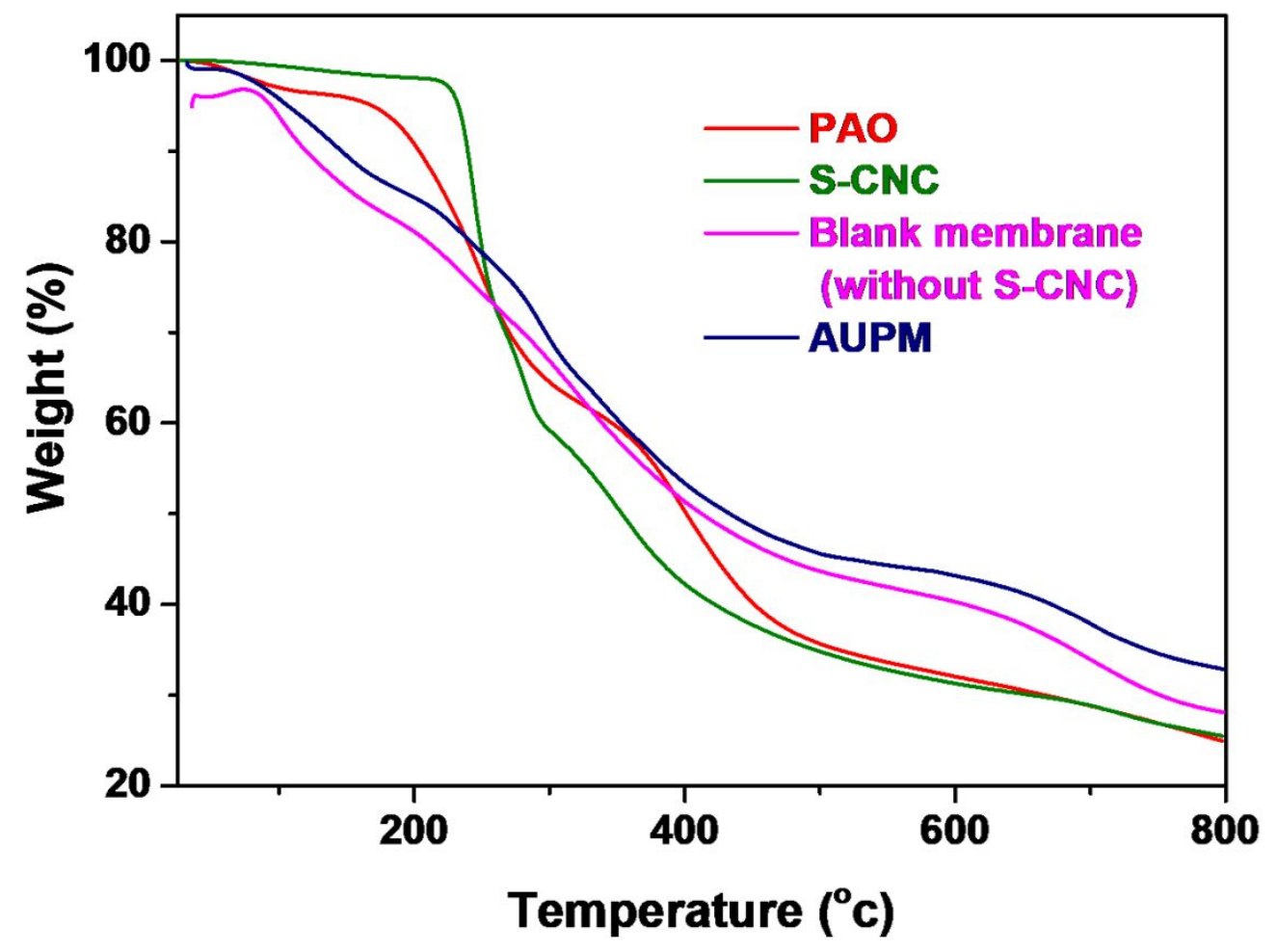

Figure S9. The TGA curves of the PAO, S-CNC, blank membrane without S-CNC and the AUPM membrane. 


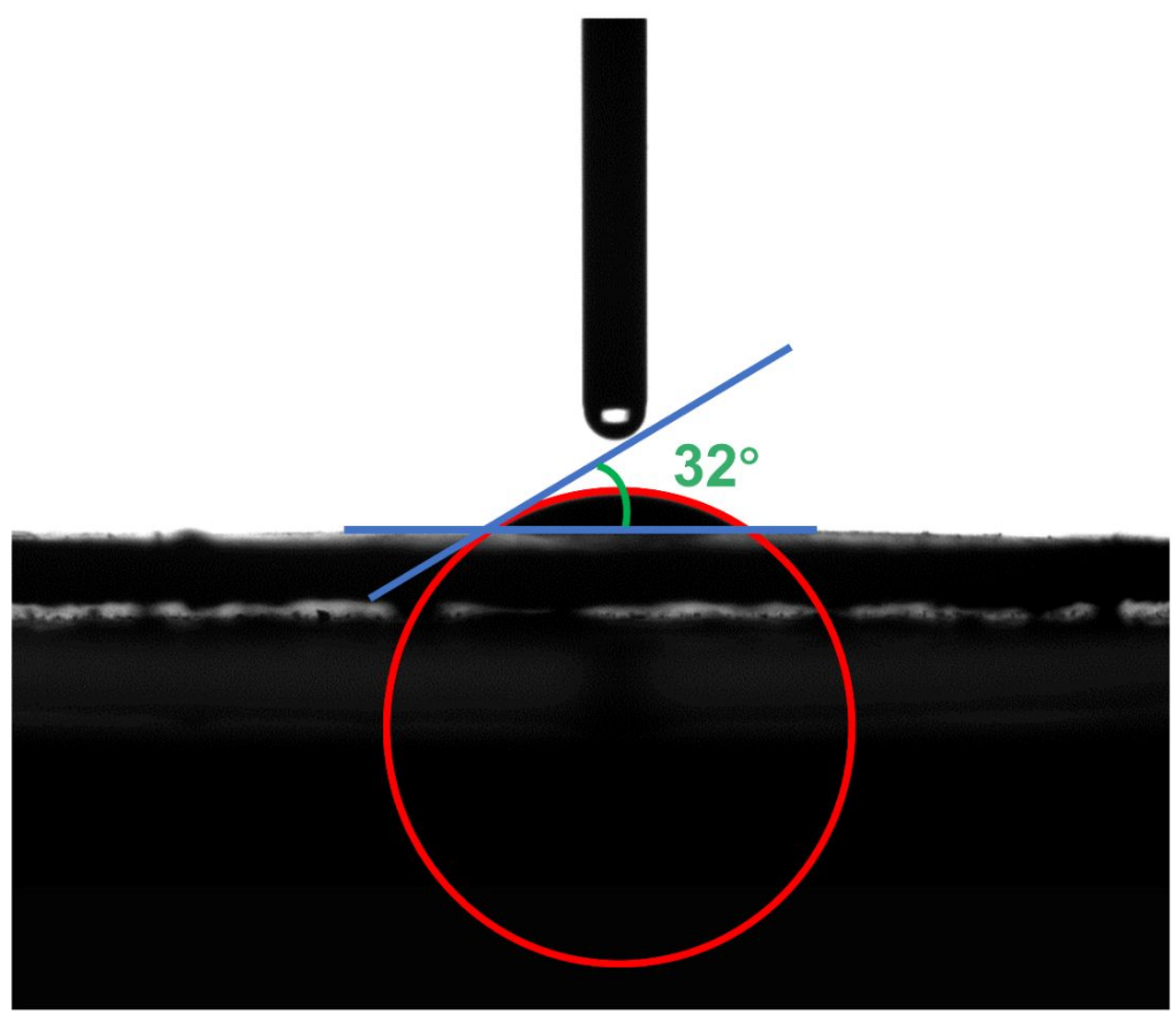

Figure S10. Illustration to detect the contact angle of non-CNC membrane, S-CNC and AUPM. 

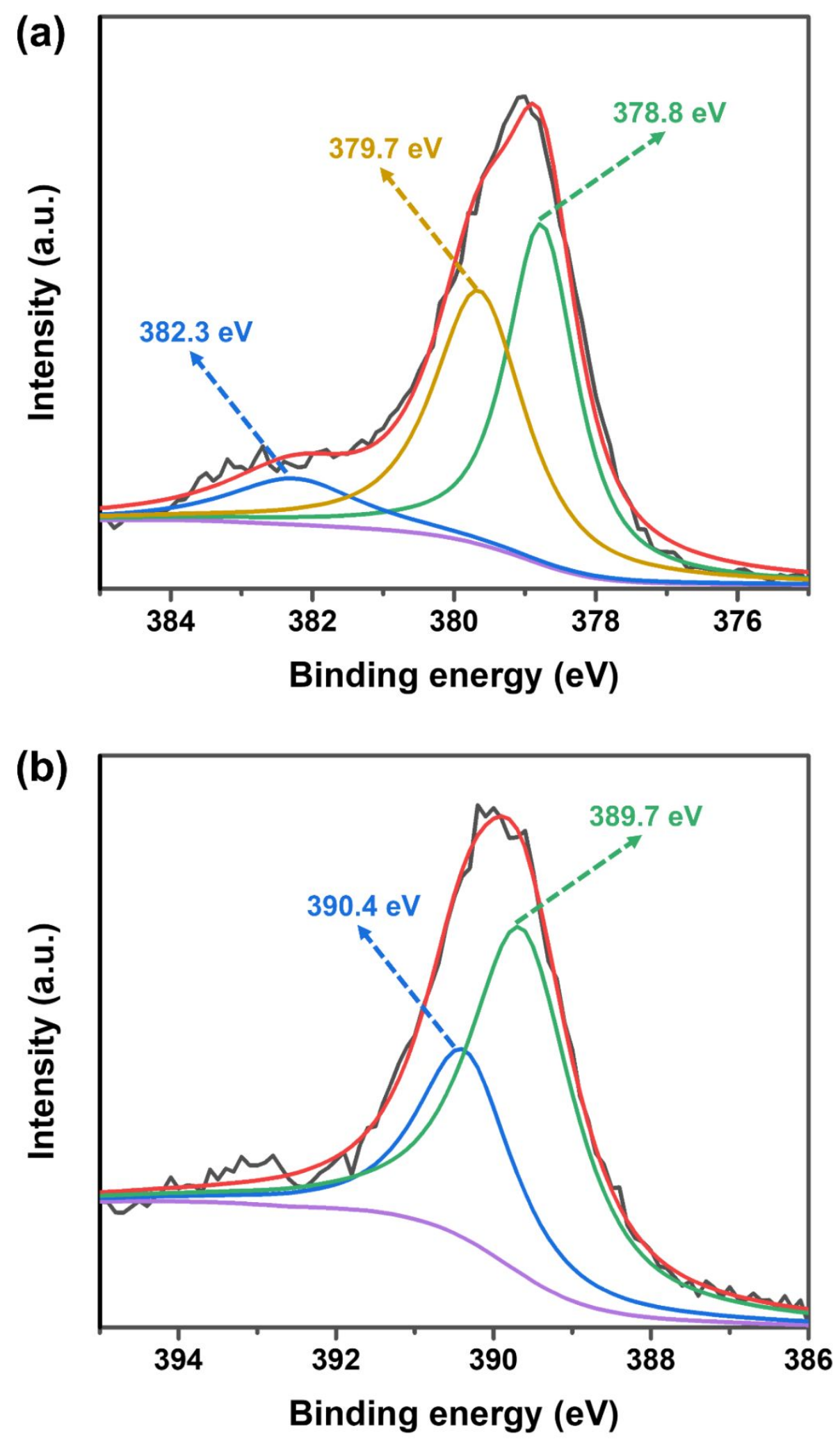

Figure S11. High-resolution XPS spectra of (a) and (b) two U4f peaks of the U-uptake AUPM. 

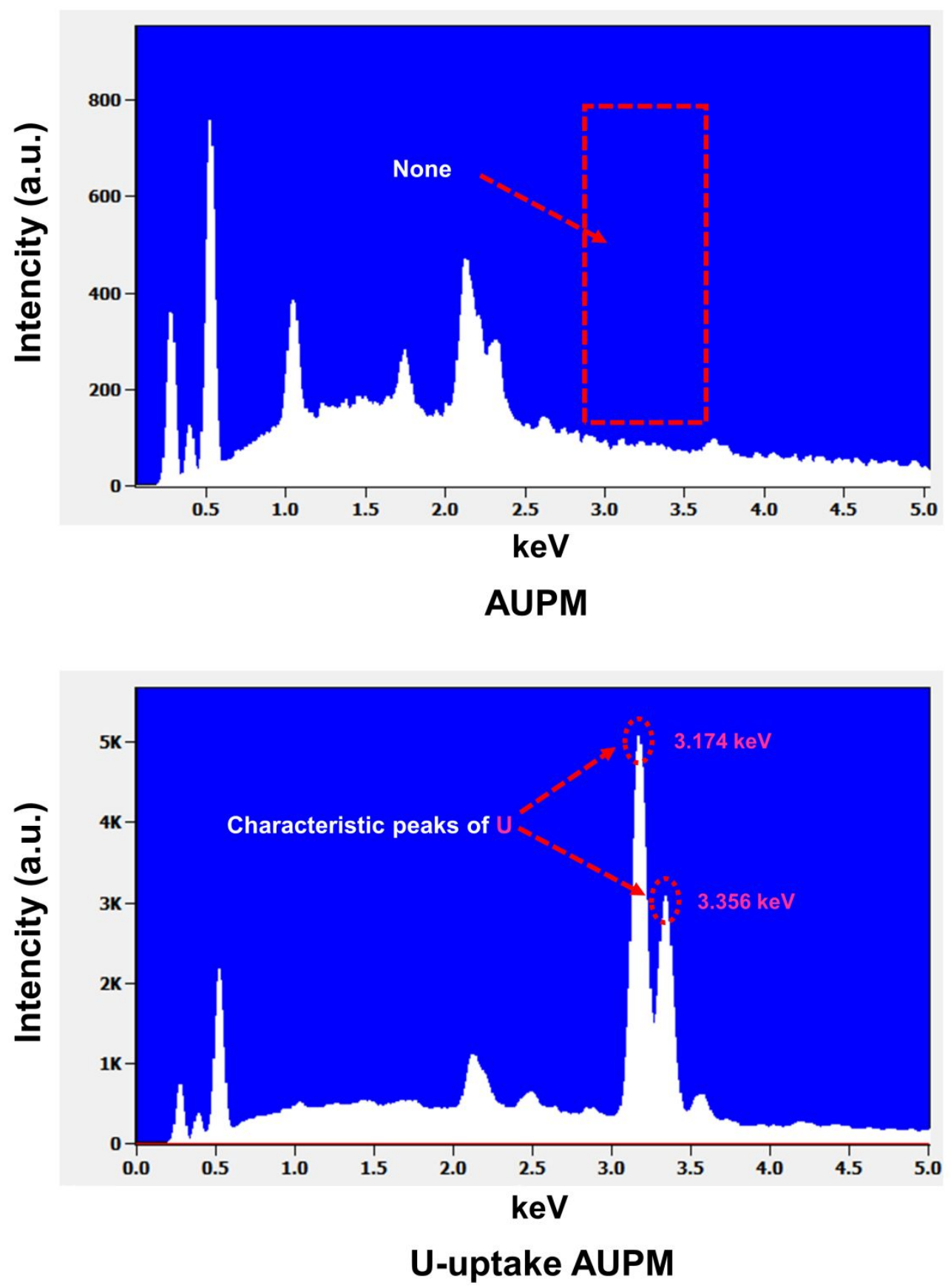

Figure S12. Comparison of the EDS mappings between the AUPM and the U-uptake AUPM. 


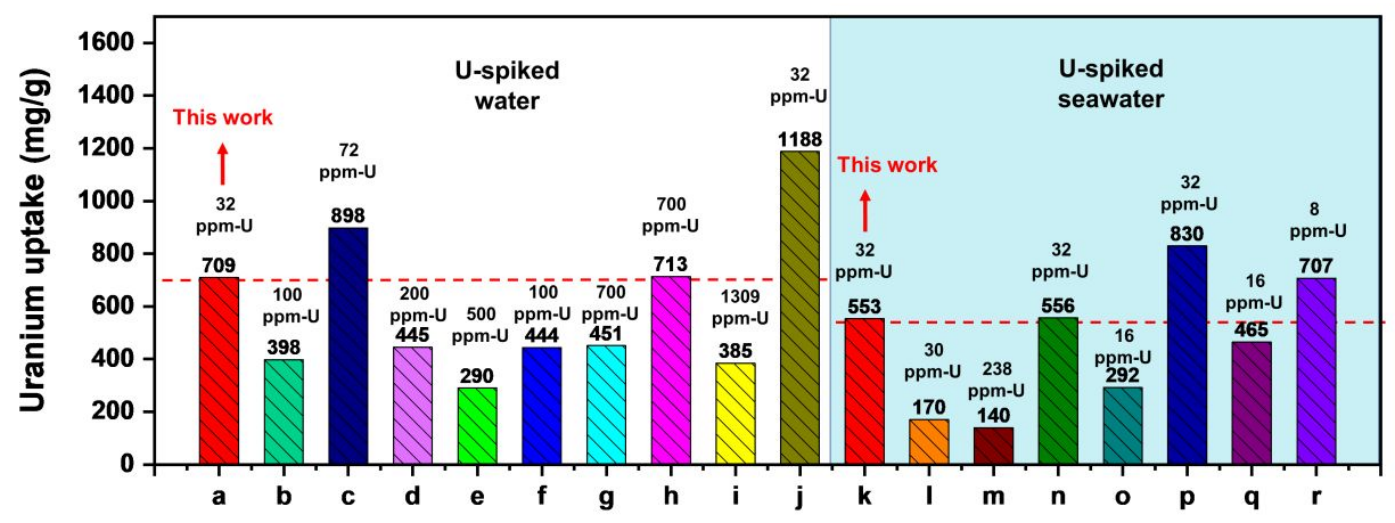

Figure S13. Comparison of the U-absorbing capacity between this AUPM and existing membrane-based U-adsorbents. ${ }^{3-13,1,14,11,15,16}$

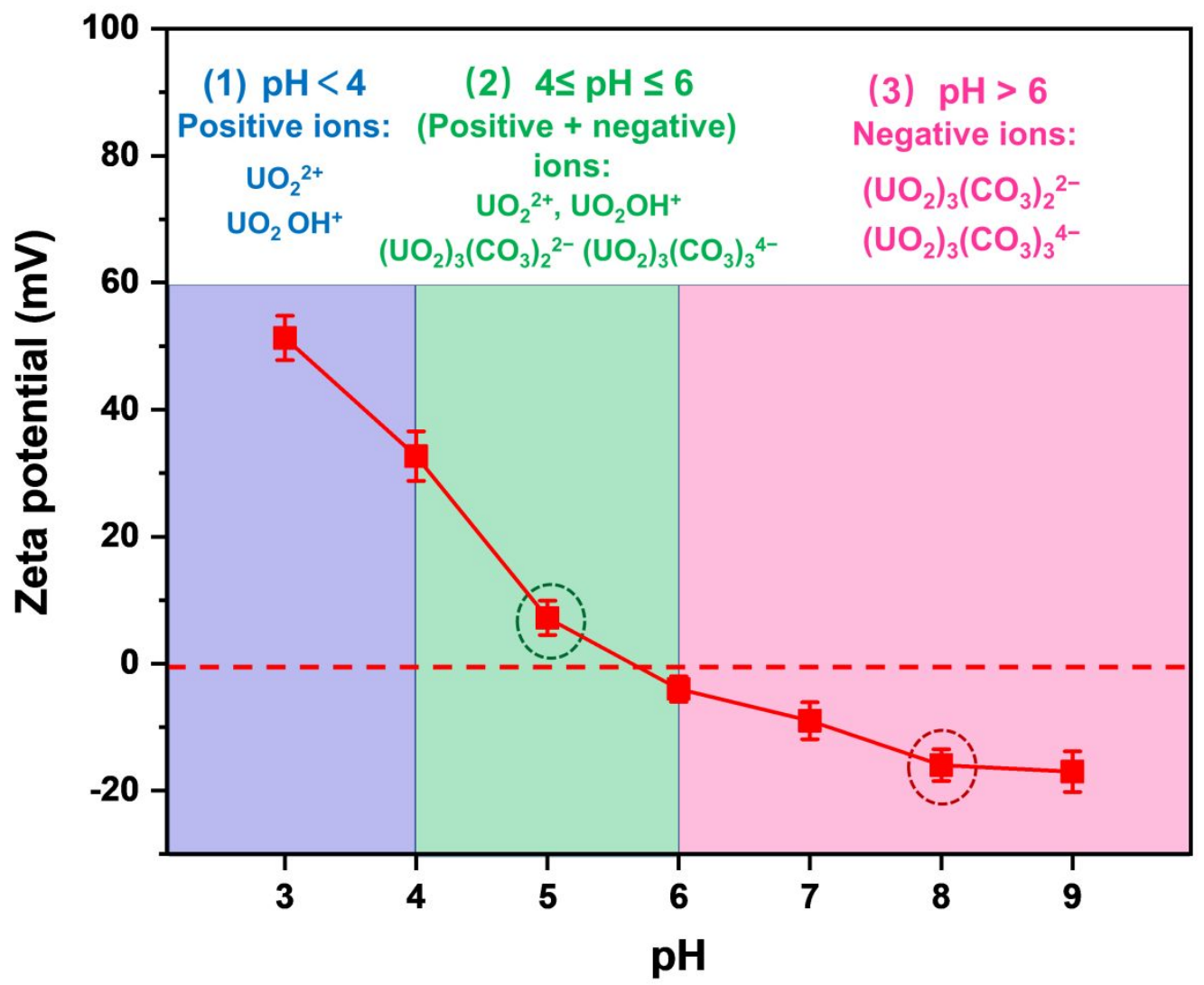

Figure S14. The the Zeta potential of the AUPM and the existent form of U(VI) at different $\mathrm{pH}$ values. 

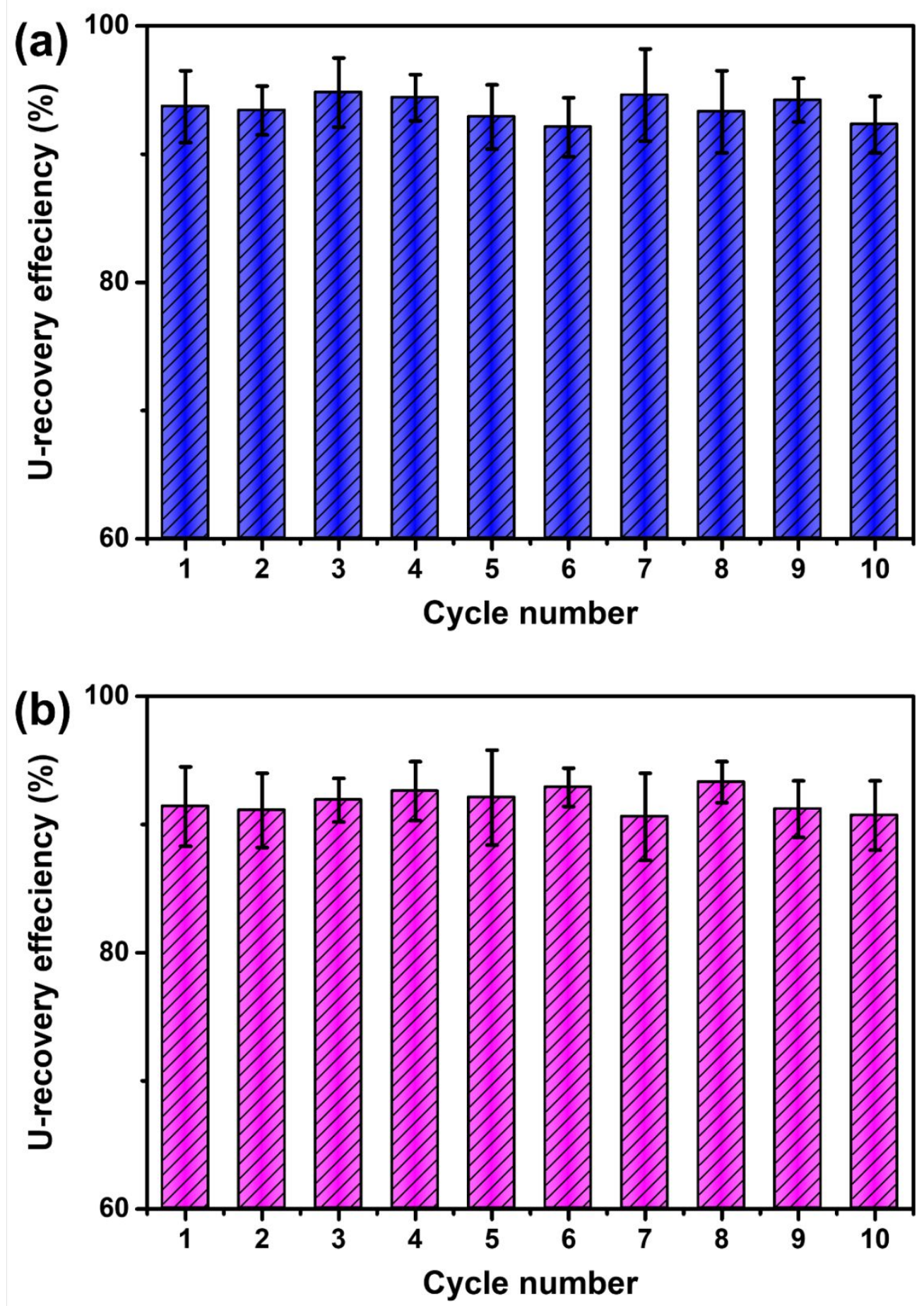

Figure S15. 10 times uranium recovery cycles of AUPM from (a) $100 \mathrm{ppb}$ U-spiked water and (b) $100 \mathrm{ppb}$ U-spiked seawater, respectively. 


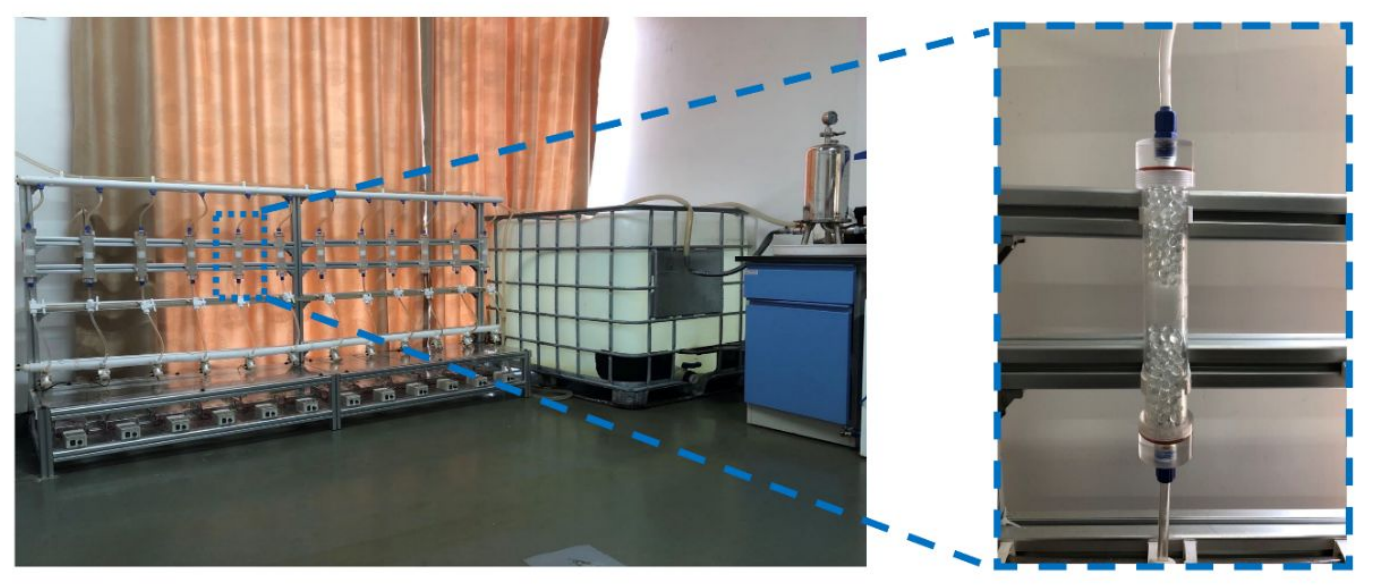

Figure S16. The seawater test system. 


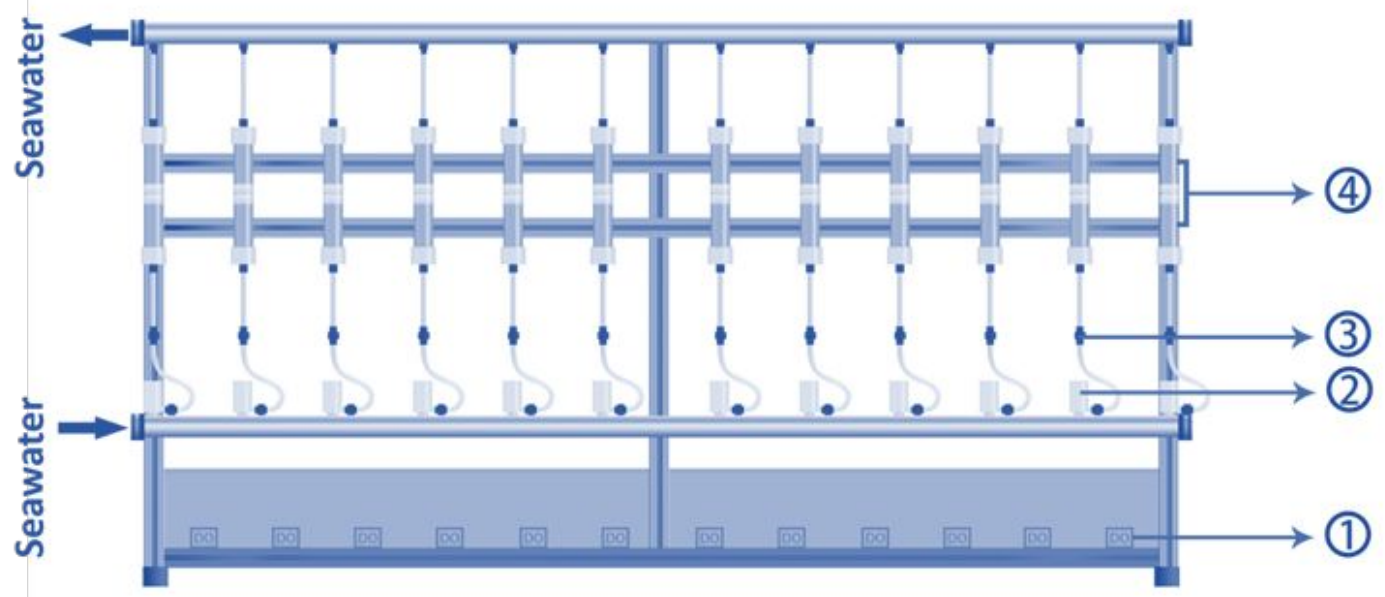

(1) Motor(flow rate) controller (2) Water pump (3) Teflon valve (4) Adsorbent bed

Figure S17. The illustration of the seawater circulation device. (1) Motor (flow rate) controller, (2) Water pump, (3) Teflon valve, (4) Adsorbent bed.
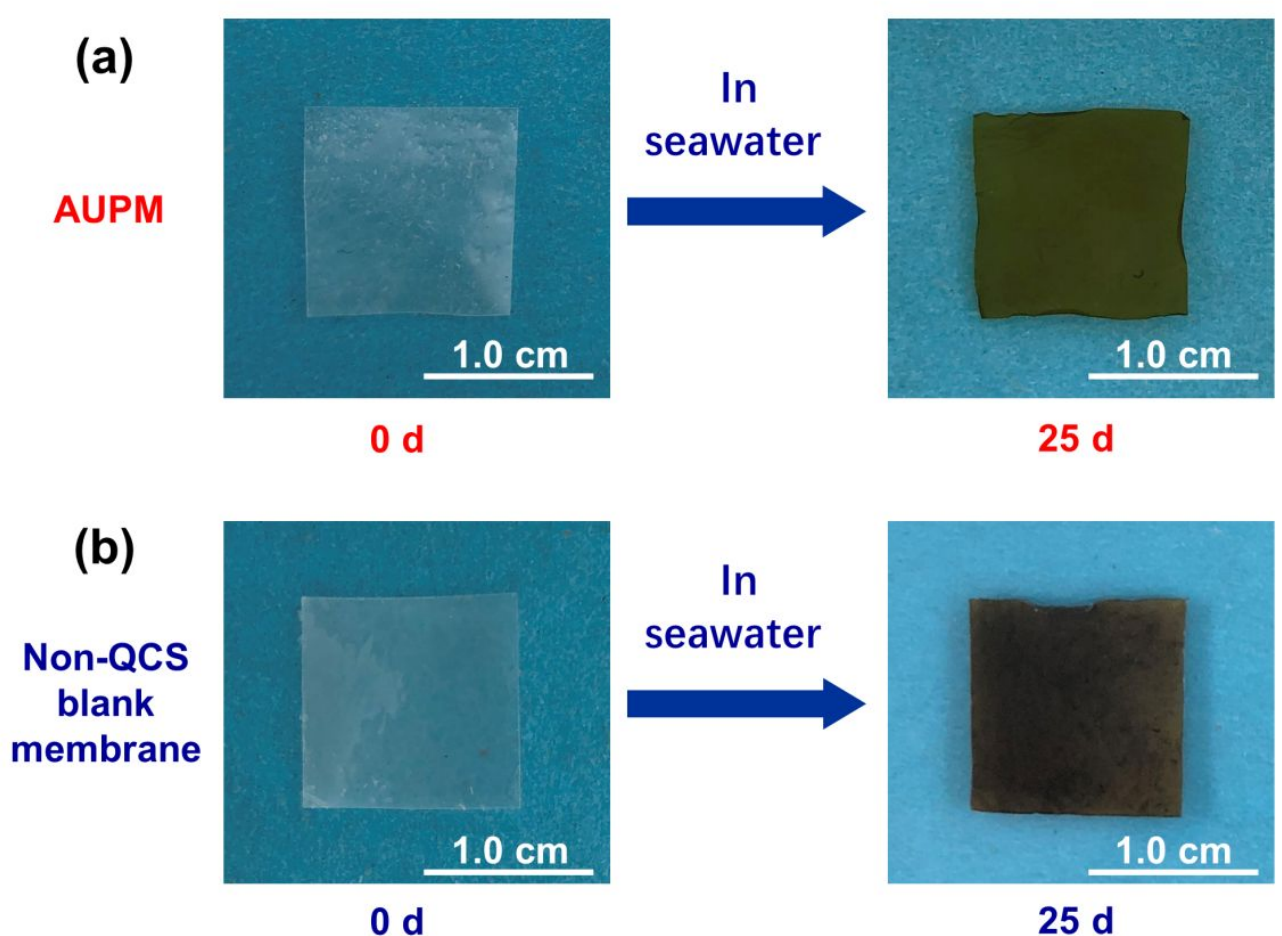

Figure S18. The color change of (a) the AUPM and (b) the Non-QCS blank membrane before 
and after 25 days in natural seawater without filtered bacteria/microorganisms.

(a)

AUPM

(b)

Non-QCS

blank membrane

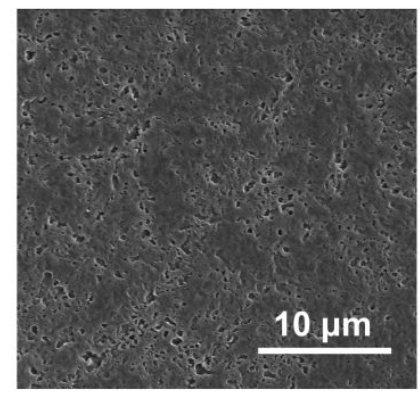

$0 \mathrm{~d}$

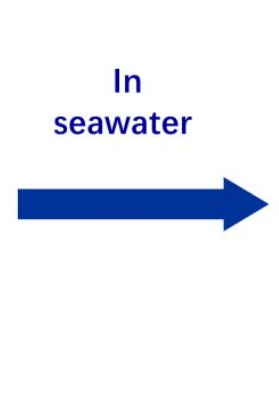

In seawater
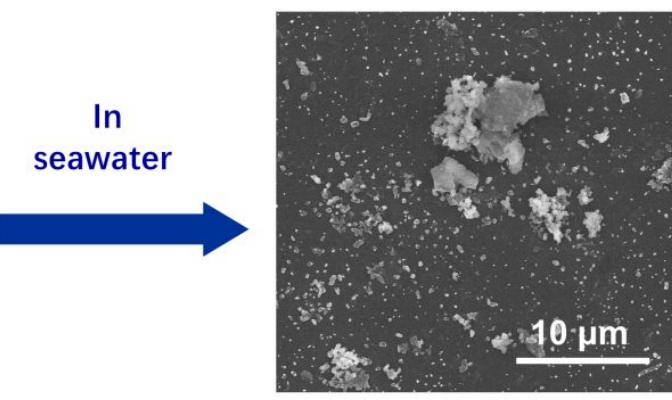

$25 \mathrm{~d}$

Figure S19. The SEM images of (a) the AUPM and (b) the Non-QCS blank membrane before and after 25 days in natural seawater without filtered bacteria/microorganisms. 
(a)

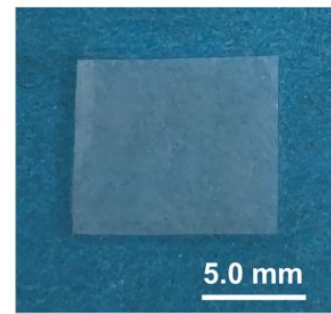

AUPM

$0 \mathrm{~d}$

(b)

Non-QCS

blank

membrane

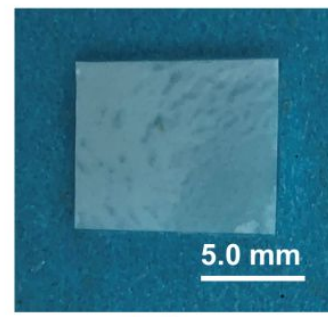

0 d

(c)

AUPM

(after 3 d)

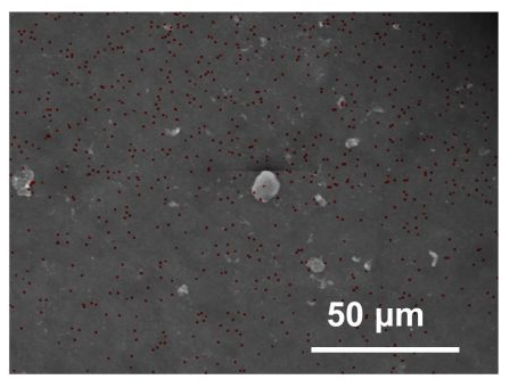

SEM

(d)

Non-QCS

blank

membrane

(after $3 \mathrm{~d}$ )

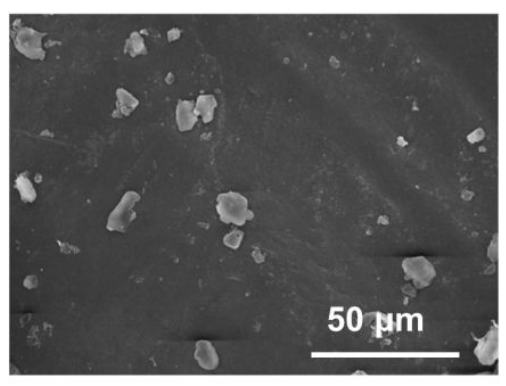

SEM
In seawater $(1.0 \mathrm{~L})$

with $100 \mathrm{mg}$ of

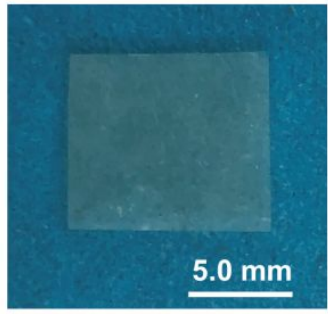

$3 \mathrm{~d}$

In seawater (1.0 L) with $100 \mathrm{mg}$ of

Polyethylene (PE)

microparticles

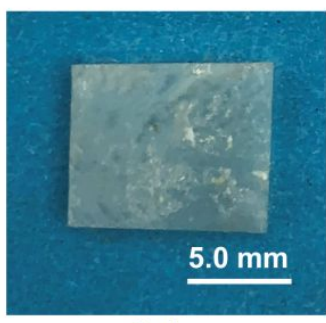

$3 \mathrm{~d}$

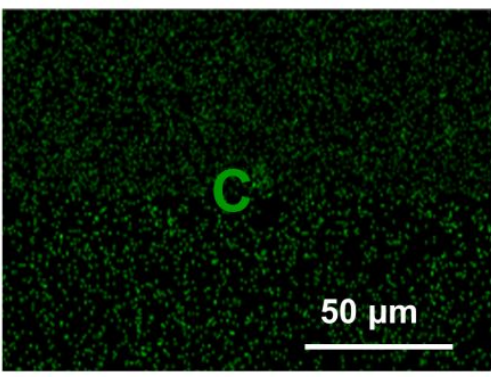

EDS mapping

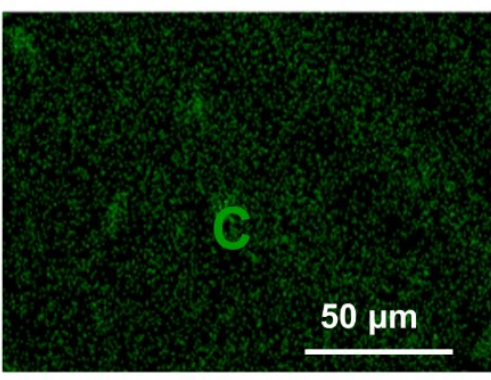

EDS mapping

Figure S20. Comparison of the anti-fouling property to polyethylene (PE) microparticles in agitated seawater between the AUPM and the non-QCS blank membrane via (a-b) photographs and (c-d) C element EDS mapping. 
(a)

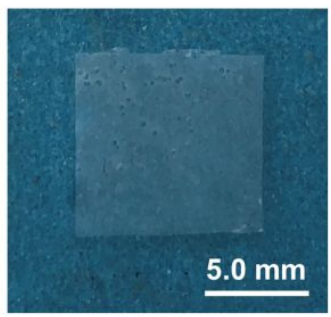

In seawater $(1.0 \mathrm{~L})$ with $100 \mathrm{mg}$ of

AUPM

od

(b)
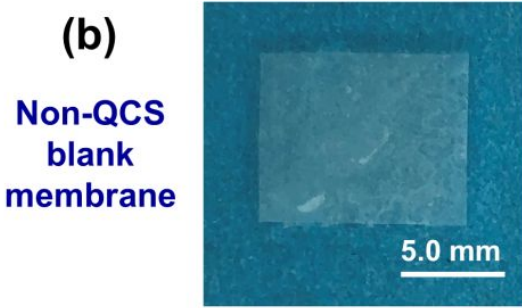

Od

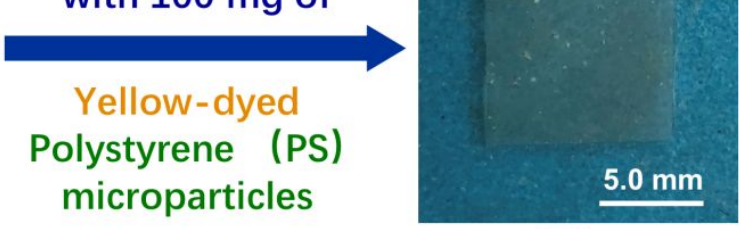

$3 \mathrm{~d}$

In seawater $(1.0 \mathrm{~L})$ with $100 \mathrm{mg}$ of

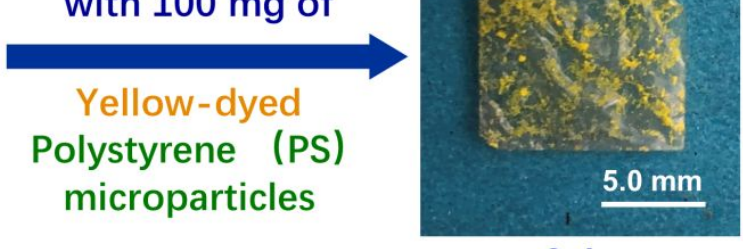

$3 \mathrm{~d}$

(c)
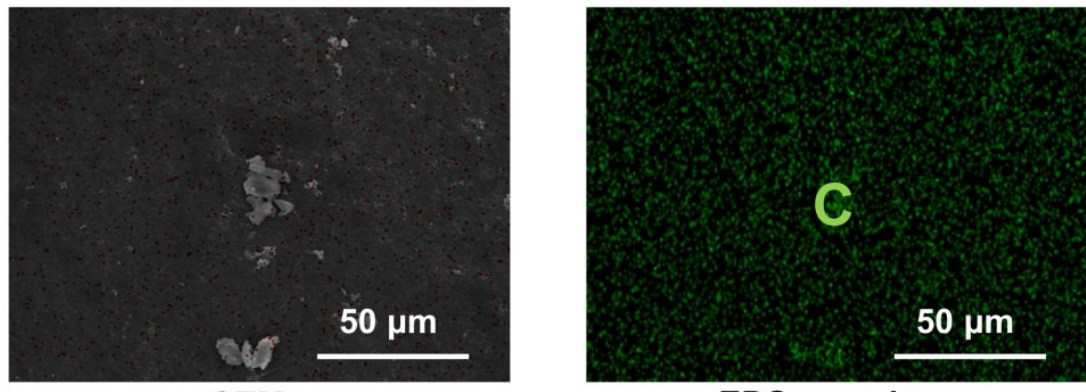

SEM

EDS mapping

(d)

Non-QCS

blank

membrane

(after 3 d)

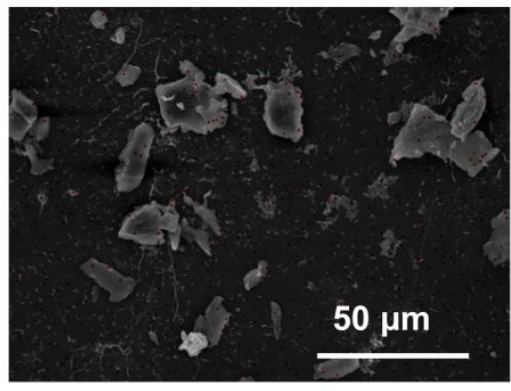

SEM

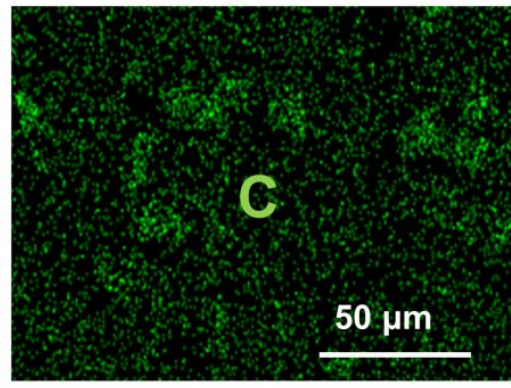

EDS mapping

Figure S21. Comparison of the anti-fouling property to yellow-dyed polystyrene (PS) microparticles in agitated seawater between the AUPM and the non-QCS blank membrane via (a-b) photographs and (c-d) C element EDS mapping. 


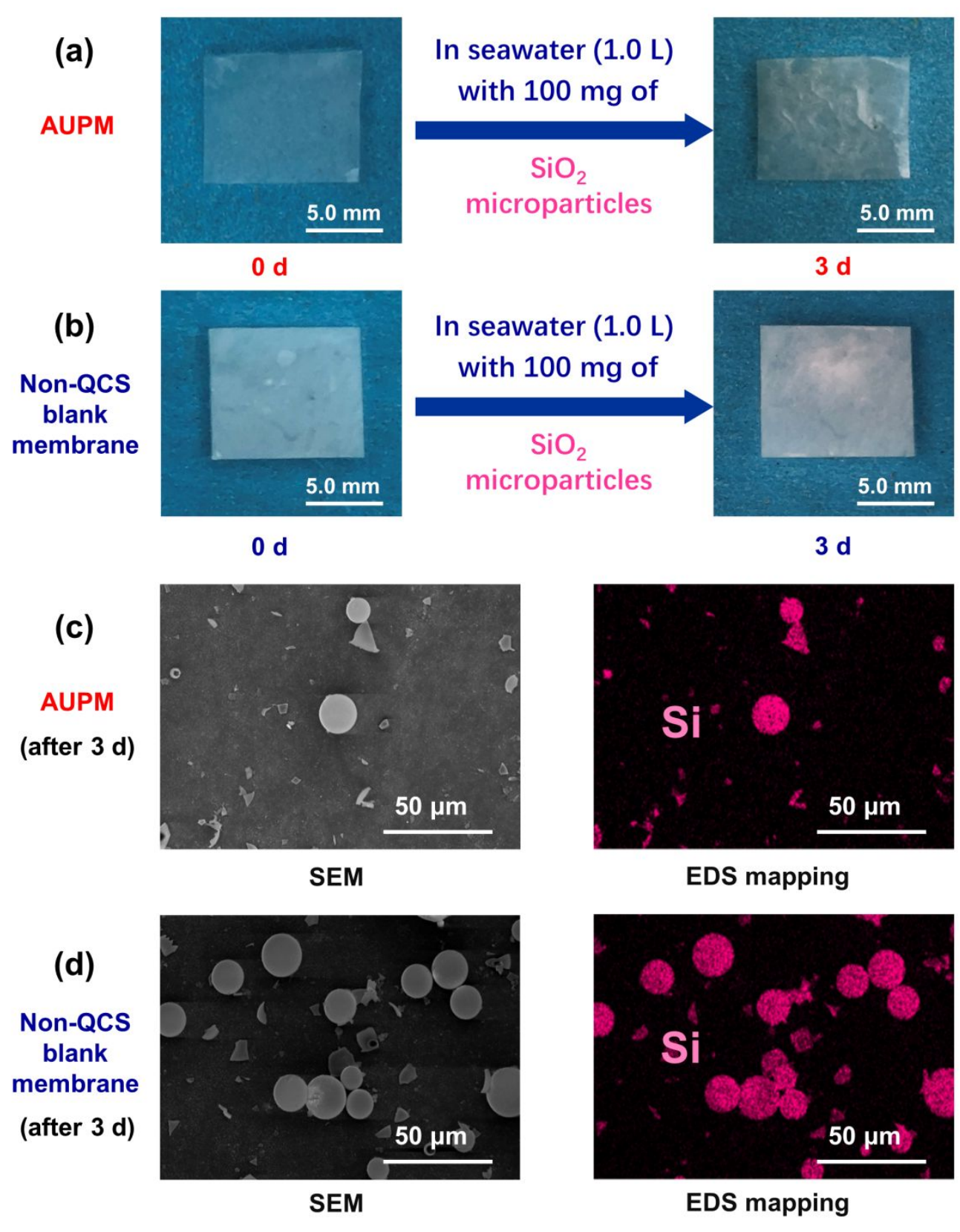

Figure S22. Comparison of the anti-fouling property to $\mathrm{SiO}_{2}$ microparticles in agitated seawater between the AUPM and the non-QCS blank membrane via (a-b) photographs and (c-d) Si element EDS mapping. 
(a)

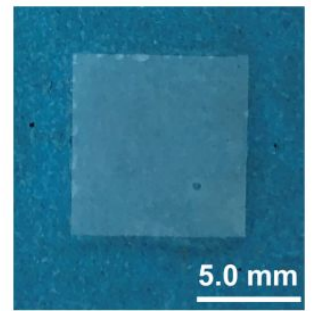

In seawater $(1.0 \mathrm{~L})$

with $100 \mathrm{mg}$ of

AUPM

O d

(b)

Non-QCS

blank

membrane

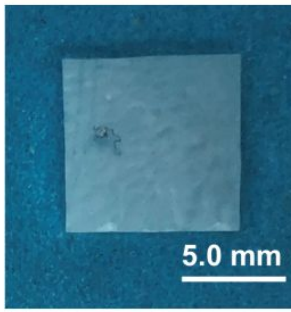

O d

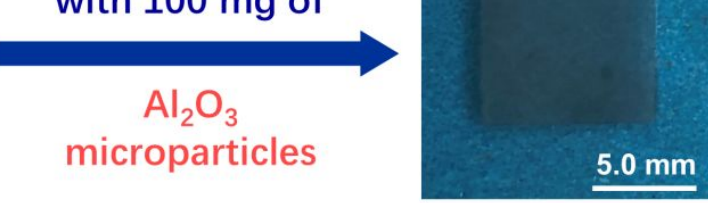

$3 \mathrm{~d}$

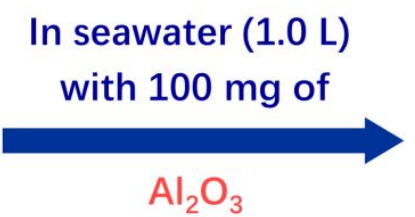

microparticles

(c)

AUPM

(after 3 d)

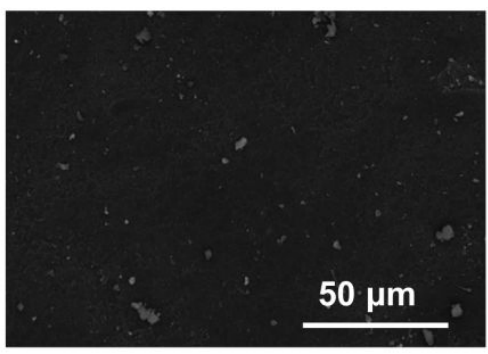

SEM

(d)

Non-QCS

blank

membrane

(after 3 d)

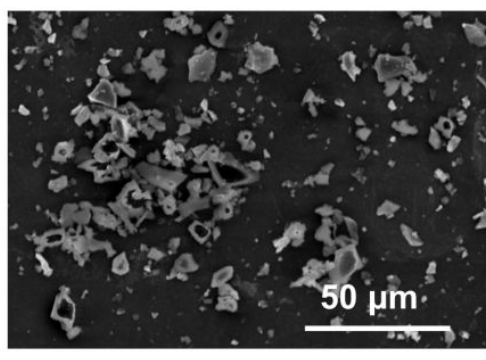

SEM
$5.0 \mathrm{~mm}$

$3 \mathrm{~d}$
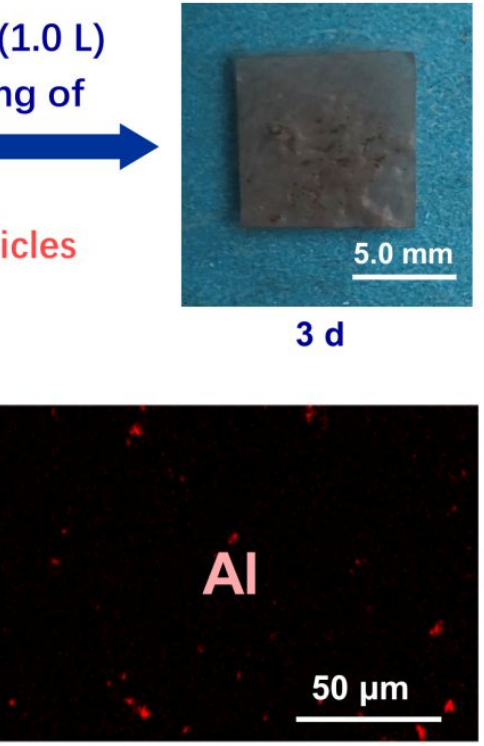

EDS mapping

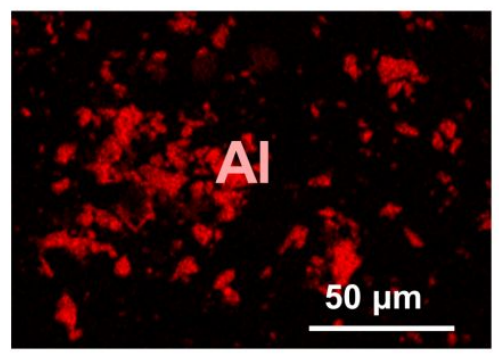

EDS mapping

Figure S23. Comparison of the anti-fouling property to $\mathrm{Al}_{2} \mathrm{O}_{3}$ microparticles in agitated seawater between the AUPM and the non-QCS blank membrane via (a-b) photographs and (cd) Al element EDS mapping. 


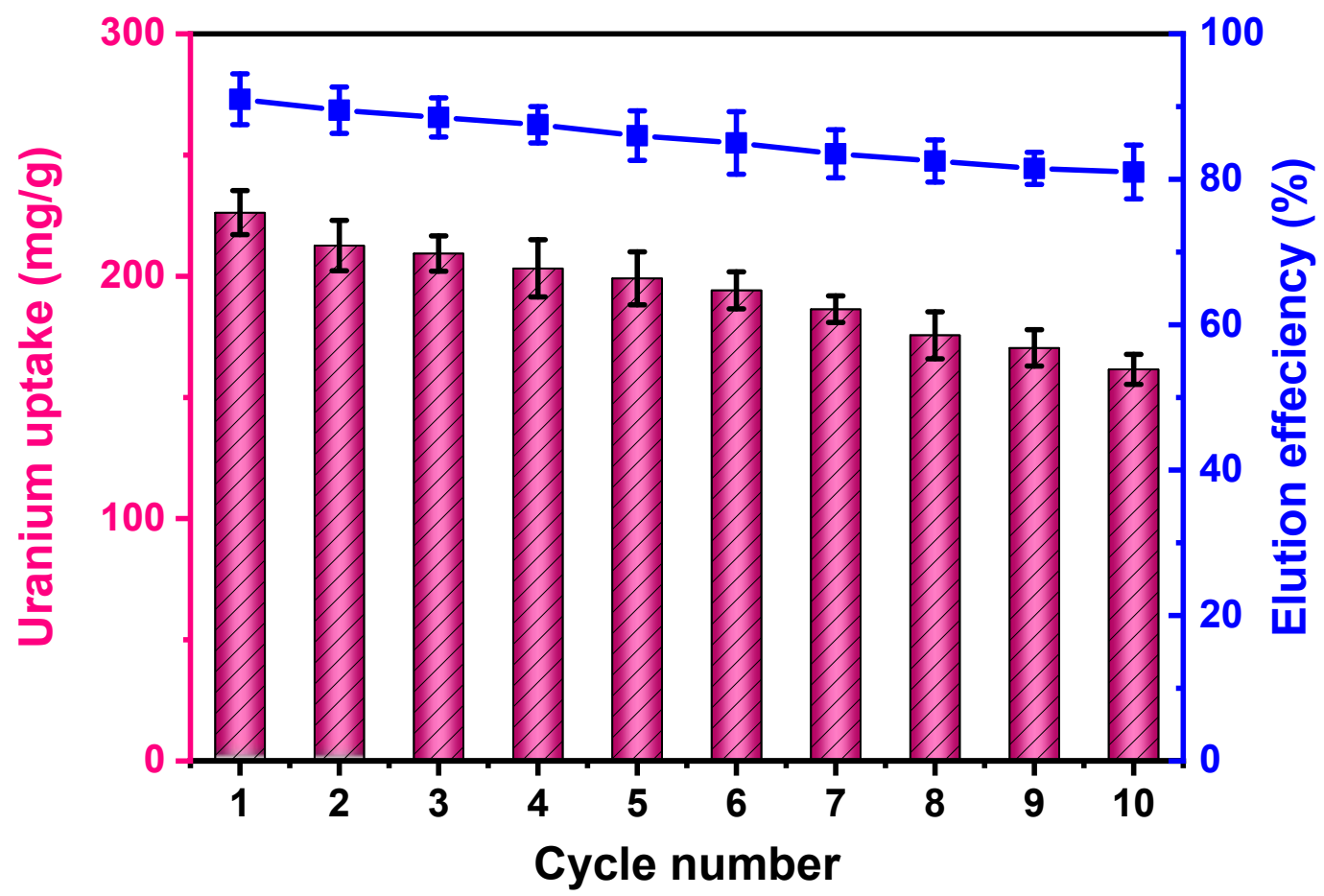

Figure S24. U-adsorbing properties (in $8 \mathrm{ppm}$ U-spiked seawater for $48 \mathrm{~h}$ ) and removal efficiencies during adsorbing-desorbing 10 cycles.

Table S1. The mass of each component in different membranes. 


\begin{tabular}{ccccc}
\hline Sample & PAO/mg & S-CNC/mg & Q-CS/mg & PAE $/ \mathrm{mg}$ \\
\hline Blank $1^{\mathrm{b}}$ & 75 & 0 & 10 & 0 \\
1 & 75 & 5 & 10 & 0.5 \\
2 & 75 & 10 & 10 & 1.0 \\
3 & 75 & 15 & 10 & 1.5 \\
Blank $2^{\mathrm{c}}$ & 75 & 15 & 10 & 0 \\
Blank $3^{\mathrm{d}}$ & 75 & 15 & 0 & 1.5 \\
4 & 75 & 20 & 10 & 2.0 \\
5 & 75 & 25 & 10 & 2.5 \\
\hline
\end{tabular}

${ }^{\mathrm{a}}$ All the samples with a $10 \pm 1 \mu \mathrm{m}$ for the uranium adsorption dumbbell shape (length: $50 \mathrm{~mm}$, width: $5 \mathrm{~mm}$, thickness: $70 \pm 5 \mu \mathrm{m}$ ) were prepared for the tensile testing. All the tests were measured at a constant rate of $50 \mathrm{~mm} / \mathrm{min}$. Blank1 is the membrane without S-CNC and PAE, blank2 is the membrane without PAE, blank3 is the membrane without Q-CS.

Table S2. Comparison of the Percentage of elements in S-CNC, Non-CNC membrane, AUPM and U-uptake AUPM through the EDS-mapping.

\begin{tabular}{ccccc}
\hline Element & $\begin{array}{c}\text { S-CNC } \\
\mathrm{wt} \%\end{array}$ & $\begin{array}{c}\text { Non-CNC } \\
\text { membrane } \\
\mathrm{wt} \%\end{array}$ & $\begin{array}{c}\text { AUPM } \\
\mathrm{wt} \%\end{array}$ & $\begin{array}{r}\text { U-uptake } \\
\text { AUPM } \\
\mathrm{Wt} \%\end{array}$ \\
\hline $\mathrm{C}$ & 14.29 & 23.78 & 16.24 & 1.23 \\
$\mathrm{~N}$ & 0 & 21.43 & 12.61 & 1.14 \\
$\mathrm{O}$ & 56.39 & 47.03 & 55.49 & 12.35 \\
$\mathrm{~S}$ & 11.85 & 0 & 0.39 & 0 \\
$\mathrm{U}$ & 0 & 0 & 0 & 85.27 \\
\hline
\end{tabular}

Table S3. The uranium adsorbing kinetic fitting parameters of AUPM in (2, 8, 16 and $32 \mathrm{ppm})$ 
U-spiked water.

\begin{tabular}{|c|c|c|c|}
\hline $\begin{array}{l}\text { Pseudo-second- } \\
\text { order model }\end{array}$ & $\mathrm{R}_{2}$ & $\mathrm{q}_{\mathrm{e}}$ & $\begin{array}{c}\mathrm{k}_{2} \\
{\left[\mathrm{~g} \mathrm{mg}^{-1} \mathrm{~min}^{-1}\right]}\end{array}$ \\
\hline $2 \mathrm{ppm}$ & 0.9998 & 172 & $7.860 \times 10^{-6}$ \\
\hline 8 ppm & 0.9998 & 385 & $4.089 \times 10^{-6}$ \\
\hline $16 \mathrm{ppm}$ & 0.9999 & 556 & $4.153 \times 10^{-6}$ \\
\hline 32 ppm & 0.9999 & 714 & $5.222 \times 10^{-6}$ \\
\hline
\end{tabular}

Table S4. The uranium adsorbing kinetic fitting parameters of AUPM in (2, 8, 16 and $32 \mathrm{ppm})$ U-spiked seawater.

\begin{tabular}{cccc}
\hline $\begin{array}{l}\text { Pseudo-second- } \\
\text { order model }\end{array}$ & $\mathrm{R}_{2}$ & $\mathrm{q}_{\mathrm{e}}$ & $\begin{array}{c}\mathrm{k}_{2} \\
{\left[\mathrm{~g} \mathrm{mg}^{-1} \mathrm{~min}^{-1}\right]}\end{array}$ \\
\hline $2 \mathrm{ppm}$ & 0.9995 & 141 & $9.505 \times 10^{-6}$ \\
& & & \\
$8 \mathrm{ppm}$ & 0.9997 & 286 & $5.430 \times 10^{-6}$ \\
& & & \\
$16 \mathrm{ppm}$ & 0.9998 & 370 & $6.434 \times 10^{-6}$ \\
& & & $6.834 \times 10^{-6}$ \\
\hline $2 \mathrm{ppm}$ & 0.9999 & 556 & \\
\hline
\end{tabular}


Table S5. Concentration of U(VI) and co-existing metal ions in seawater and $100 \times$ seawater (the $\mathrm{Na}, \mathrm{K}, \mathrm{Ca}$ and $\mathrm{Mg}$ macro-elements was the same as that in seawater). ${ }^{17}$

\begin{tabular}{|c|c|c|c|}
\hline Element & $\begin{array}{l}\text { Con. In natural } \\
\qquad \mathrm{SW} / \mathrm{ppb}\end{array}$ & $\begin{array}{l}\text { Con. In } 100 \times \mathrm{SW} \\
\text { (calculated)/ppb }\end{array}$ & Ions \\
\hline $\mathrm{U}$ & 3.3 & 330 & $\mathrm{U}(\mathrm{VI})$ \\
\hline $\mathrm{V}$ & $1.5-2.5$ & 200 & $\mathrm{~V}(\mathrm{~V})$ \\
\hline $\mathrm{Fe}$ & $1.0-2.0$ & 150 & $\mathrm{Fe}^{3+}$ \\
\hline Co & 0.05 & 5.0 & $\mathrm{Co}^{2+}$ \\
\hline $\mathrm{Ni}$ & 1 & 100 & $\mathrm{Ni}^{2+}$ \\
\hline $\mathrm{Cu}$ & 0.6 & 60 & $\mathrm{Cu}^{2+}$ \\
\hline $\mathrm{Cr}$ & 0.5 & 50 & $\mathrm{Cr}^{3+}$ \\
\hline $\mathrm{Ba}$ & 0.2 & 20 & $\mathrm{Ba}^{2+}$ \\
\hline $\mathrm{Mn}$ & 1.3 & 130 & $\mathrm{Mn}^{2+}$ \\
\hline $\mathrm{Na}$ & $9.56 \times 10^{6}$ & $9.56 \times 10^{6}$ & $\mathrm{Na}+$ \\
\hline K & $0.653 \times 10^{6}$ & $0.653 \times 10^{6}$ & $\mathrm{~K}+$ \\
\hline $\mathrm{Ca}$ & $0.873 \times 10^{6}$ & $0.873 \times 10^{6}$ & $\mathrm{Ca} 2+$ \\
\hline $\mathrm{Mg}$ & $1.22 \times 10^{6}$ & $1.22 \times 10^{6}$ & $\mathrm{Mg} 2+$ \\
\hline
\end{tabular}


Table S6. Comparison of the uranium adsorption performance from nature seawater between the AUPM and many other uranium adsorbents.

\begin{tabular}{|c|c|c|c|c|c|}
\hline Absorbents & $\begin{array}{l}\mathrm{U}_{\text {adsorbent }} \\
\left(\mathrm{mg} \cdot \mathrm{g}^{-1}\right)\end{array}$ & $\begin{array}{l}\text { Time } \\
\text { (day) }\end{array}$ & $\begin{array}{c}U_{\text {adsorbent }} \\
\text { Average rate } \\
\left(\mathrm{mg} \cdot \mathrm{g}^{-1} \cdot \text { day }^{-1}\right)\end{array}$ & Year & Ref. \\
\hline This work & 8.78 & 25 & 0.351 & & \\
\hline PAO membrane & 9.35 & 35 & 0.267 & 2020 & [16] \\
\hline AO-aerogel & 9.29 & 30 & 0.31 & 2020 & [18] \\
\hline PAO hydrogel & 4.87 & 28 & 0.174 & 2019 & [1] \\
\hline $\begin{array}{l}\text { PAO hydrogel- } \\
\text { sponge hybrid }\end{array}$ & 1.87 & 56 & 0.033 & 2019 & [14] \\
\hline PAO hydrogel & 3.9 & 28 & 0.139 & 2020 & {$[15]$} \\
\hline PAO hygrogel & 9.23 & 28 & 0.33 & 2020 & [11] \\
\hline $\begin{array}{r}\text { MOF } \\
\text { hydrogel }\end{array}$ & 6.99 & 35 & 0.200 & 2020 & [19] \\
\hline $\begin{array}{l}\text { AO -based } \\
\text { fiber }\end{array}$ & 6 & 60 & 0.100 & 2001 & [20] \\
\hline $\mathrm{AO}$-based fiber & 2 & 60 & 0.033 & 2006 & [21] \\
\hline AO-based fiber & 1.5 & 30 & 0.050 & 2010 & [22] \\
\hline $\mathrm{AO}$-based fiber & 2 & 30 & 0.067 & 2013 & [23] \\
\hline $\mathrm{AO}$-based fiber & 3.3 & 56 & 0.059 & 2014 & [24] \\
\hline $\begin{array}{l}\text { polyethylene } \\
\text { fiber }\end{array}$ & 5.22 & 49 & 0.107 & 2016 & [25] \\
\hline $\mathrm{AO}$-based fiber & 3.5 & 56 & 0.063 & 2016 & [26] \\
\hline $\begin{array}{l}\text { polyethylene } \\
\text { fiber }\end{array}$ & 7.4 & 96 & 0.077 & 2017 & [27] \\
\hline $\begin{array}{l}\text { AO-based } \\
\text { nanofiber }\end{array}$ & 8.7 & 56 & 0.155 & 2018 & [17] \\
\hline $\mathrm{AO}$-based fiber & 11.5 & 90 & 0.128 & 2019 & [28] \\
\hline AO-based nanofiber & 9.59 & 56 & 0.171 & 2019 & [29] \\
\hline AO-based nanofiber & 1.14 & 40 & 0.029 & 2020 & [30] \\
\hline AO-based nanofiber & 10.31 & 35 & 0.295 & 2020 & [31] \\
\hline
\end{tabular}


Table S7. Uranium extraction capability of AUPM during 10 times of recycling.

\begin{tabular}{|c|c|c|c|}
\hline Cycles & $\begin{array}{l}\text { Capacity in } 8 \text { ppm U- } \\
\text { added seawater }\left(\mathrm{mg} \cdot \mathrm{g}^{-1}\right)\end{array}$ & $\begin{array}{l}\text { Ratio to initial } \\
\text { capacity (\%) }\end{array}$ & $\begin{array}{l}\text { Capacity in natural } \\
\text { seawater }\left(\mathrm{mg} \cdot \mathrm{g}^{-1}\right)\end{array}$ \\
\hline 1 & 226.25 & 100 & 8.78 \\
\hline 2 & 212.64 & 93.98 & 8.25 \\
\hline 3 & 209.35 & 92.53 & 8.12 \\
\hline 4 & 203.26 & 89.84 & 7.89 \\
\hline 5 & 199.13 & 88.01 & 7.73 \\
\hline 6 & 194.17 & 85.82 & 7.53 \\
\hline 7 & 186.39 & 82.38 & 7.23 \\
\hline 8 & 175.64 & 77.63 & 6.82 \\
\hline 9 & 170.43 & 75.33 & 6.61 \\
\hline 10 & 161.56 & 71.41 & 6.27 \\
\hline Total & / & 1 & 75.23 \\
\hline
\end{tabular}


Table S8. Economic cost of manufacturing 1 ton of AUPM from industrial raw materials.

\begin{tabular}{cccc}
\hline Raw material & Price per unit & Quantity (ton) & Total price \\
\hline PAN & $\$ 3060 /$ ton & 0.625 & $\$ 1912.5$ \\
$\mathrm{NH}_{2} \mathrm{OH} \cdot \mathrm{HCL}$ & $\$ 580 /$ ton & 0.875 & $\$ 507.5$ \\
$\mathrm{Na}_{2} \mathrm{CO}_{3}$ & $\$ 150 /$ ton & 0.625 & $\$ 93.75$ \\
$\mathrm{NaOH}$ & $\$ 260 /$ ton & 0.5 & $\$ 130$ \\
$\mathrm{DMF}$ & $\$ 580 /$ ton & 6.25 & $\$ 3625$ \\
$\mathrm{MCC}$ & $\$ 1230 /$ ton & 0.21 & $\$ 258.3$ \\
$\mathrm{H}_{2} \mathrm{SO}$ & $\$ 45 /$ ton & 1.837 & $\$ 82.665$ \\
$\mathrm{PAE}$ & $\$ 305 /$ ton & 0.015 & $\$ 4.575$ \\
$\mathrm{Q}-\mathrm{CS}$ & $\$ 18370 /$ ton & 0.1 & $\$ 1837$ \\
Total & / & $\$ 8451.29$ \\
\hline
\end{tabular}

Table S9. The operation cost of extracting uranium from seawater for 10 times per ton of adsorbent.

\begin{tabular}{cccc}
\hline Operation & Price per unit & Quantity & Total price \\
\hline Gauze bag & $\$ 4.6 / \mathrm{m}^{3}$ & $6 \mathrm{~m}^{3}$ & $\$ 27.6$ \\
Energy cost & $\$ 60 /$ time & 10 times & $\$ 600$ \\
Labor cost & $\$ 235 /$ time & 10 times & $\$ 2350$ \\
Ship & $\$ 55 /$ time & 10 times & $\$ 550$ \\
Other consumables & $\$ 60 /$ time & 10 times & $\$ 600$ \\
(Cage, rope, et al. $)$ & & & $\$ \mathbf{4 1 2 7 . 6 0}$ \\
\hline
\end{tabular}




\section{Supporting Movies}

Movie S1. The demonstration of the AUPM mechanical strength.

Movie S2. U-adsorption process of the AUPM in U-spiked water.

Movie S3. U-desorption process of the AUPM in elution solution.

\section{References}

(1) Ma, C. X.; Gao, J. X.; Wang, D.; Yuan, Y. H.; Wen, J.; Yan, B. J.; Zhao, S. L.; Zhao, X. M.; Sun, Y.; Wang, X. L.; Wang, N. Sunlight Polymerization of Poly(amidoxime) Hydrogel Membrane for Enhanced Uranium Extraction from Seawater. Adv. Sci. 2019, 6(13), 1900085.

(2) Pan, H.-B.; Liao, W.; Wai, C. M.; Oyola, Y.; Janke, C. J.; Tian, G.; Rao, L. Carbonate- $\mathrm{H}_{2} \mathrm{O}_{2}$ Leaching for Sequestering Uranium from Seawater. Dalton Trans. 2014, 43 (28), 10713-10718.

(3) Wang, F. H.; Li, H. P.; Liu, Q.; Li, Z. S.; Li, R. M.; Zhang, H. S.; Liu, L.H.; Emelchenko, G. A.; Wang, J. A Graphene Oxide/Amidoxime Hydrogel for Enhanced Uranium Capture. Sci. Rep. 2016, 6, 19367.

(4) Wang, X.; Liu, Q.; Liu, J. Y.; Chen, R. R.; Zhang, H. S.; Li, R. M.; Li, Z. S.; Wang, J. 3D SelfAssembly Polyethyleneimine Modified Graphene Oxide Hydrogel for the Extraction of Uranium from Aqueous Solution. Appl. Surf. Sci. 2017, 426, 1063-1074.

(5) Yi, X. F.; Xu, Z. Q.; Liu, Y.; Guo, X. Y.; Qu, M. R.; Xu, X. P. Highly efficient removal of uranium(VI) from wastewater by polyacrylic acid hydrogels. $R S C A d v .2017,7,6278-6287$.

(6) He, J. R.; Sun, F. L.; Han, F. H.; Gu, J. J.; Qu, M. R.; Xu, W. K.; Xu, X. P. Preparation of a Novel Polyacrylic Acid and Chitosan Interpenetrating Network Hydrogel for Removal of U(VI) from Aqueous Solutions. RSC Adv. 2018, 8, 12684.

(7) Su, S. Z.; Chen, R. R.; Liu, Q.; Liu, J. Y.; Zhang, H. S.; Li, R. M.; Zhang, M. L.; Liu, P. L.; Wang, J. High Efficiency Extraction of U(VI) from Seawater by Incorporation of Polyethyleneimine, Polyacrylic Acid Hydrogel and Luffa Cylindrical Fibers. Chem. Eng. J. 2018, $345,526-535$ 
(8) He, J. R.; Jin, J. L.; Wang, Z. Z.; Yin, H. W.; Wei, C. C.; Xu, X. P. Encapsulating Nanosilica into Polyacrylic Acid and Chitosan Interpenetrating Network Hydrogel for Preconcentration of Uranium from Aqueous Solutions. J. Radioanal. Nucl. Chem. 2018, 317, 1299-1309.

(9) Wei, C. C.; Yang, M. X.; Guo, Y. Y.; Xu. W. K.; Gu, J. J.; Qu, M. R.; Xu, X. P. Highly Efficient Removal of Uranium(VI) from Aqueous Solutions by Poly(acrylic acid-co-acrylamide) Hydrogels. J. Radioanal. Nucl. Chem. 2018, 315, 211-221.

(10) Moghaddama, R. H.; Dadfarnia, S.; Shabani, A. M. H.; Tavakol, M. Synthesis of Composite Hydrogel of Glutamic Acid, Gum Tragacanth, and Anionic Polyacrylamide by Electron Beam Irradiation for Uranium (VI) Removal from Aqueous Samples: Equilibrium, Kinetics, and Thermodynamic Studies. Carbohyd. Polym. 2019, 206, 352-361.

(11) Yan, B. J.; Ma, C. X.; Gao, J. X.; Yuan, Y. H.; Wang, N. An Ion-Crosslinked Supramolecular Hydrogel for Ultrahigh and Fast Uranium Recovery from Seawater. Adv. Mater. 2020, 32(10), 1906615.

(12) Zhou, L. M.; Zou, H. B.; Wang, Y.; Liu, Z. Y.; Le, Z. G.; Huang, G. L.; Luo, T. A.; Adesina, A. A. Immobilization of in Situ Generated $\mathrm{Fe}^{0}$-Nanoparticles in Tripolyphosphate-Crosslinking Chitosan Membranes for Enhancing U(VI) Adsorption. J. Radioanal. Nucl. Chem. 2017, 311, 779-787.

(13) Luo, W.; Xiao, G.; Tian, F.; Richardson, J. J.; Wang, Y. P.; Zhou, J. F.; Guo, J. L.; Liao, X. P.; Shi, B. Engineering Robust Metal-Phenolic Network Membranes for Uranium Extraction from Seawater. Energy Environ. Sci. 2019, 12 (2), 607-614.

(14) Wang, D.; Song, J.; Lin, S.; Wen, J.; Ma, C.; Yuan, Y.; Lei, M.; Wang, X.; Wang, N.; Wu, H. A Marine-Inspired Hybrid Sponge for Highly Efficient Uranium Extraction from Seawater. $A d v$. Funct. Mater. 2019, 29(28), 1901009.

(15) Gao, J. X.; Yuan, Y. H.; Yu, Q. H.; Yan, B. J.; Qian, Y. X.; Wen, J.; Ma, C. X.; Jiang, S. H.; Wang, X. L.; Wang, N. Bio-Inspired Antibacterial Cellulose Paper-Poly(amidoxime) Composite Hydrogel for Highly Efficient Uranium(VI) Capture from Seawater. Chem. Commun. 2020, 56(28), $3935-3938$. 
(16) Shi, S.; Qian, Y.; Mei, P.; Yuan, Y.; Jia, N.; Dong, M.; Fan, J; Guo, Z.; Wang, N. Robust Flexible Poly(amidoxime) Porous Network Membranes for Highly Efficient Uranium Extraction from Seawater. Nano Energy. 2020, 71, 104629.

(17) Wang, D.; Song, J.; Wen, J.; Yuan, Y.; Liu, Z.; Lin, S.; Wang, H.; Wang, H.; Zhao, S.; Zhao, X.; Fang, M.; Lei, M.; Li, B.; Wang, N.; Wang, X.; Wu, H. Significantly Enhanced Uranium Extraction from Seawater with Mass Produced Fully Amidoximated Nanofiber Adsorbent. $A d v$. Energy Mater. 2018, 8 (33), 1802607.

(18) Shi, S.; Li, B. C.; Qian, Y. X.; Mei, P. P.; Wang. N. A Simple and Universal Strategy to Construct Robust and Anti-Biofouling Amidoxime Aerogels for Enhanced Uranium Extraction from Seawater. Chem. Eng. J. 2020, 397, 125337.

(19) Bai, Z.; Liu, Q.; Zhang, H.; Yu, J.; Chen, R.; Liu, J.; Song, D.; Li, R.; Wang, J. Anti-Biofouling and Water-Stable Balanced Charged Metal Organic Framework-Based Polyelectrolyte Hydrogels for Extracting Uranium from Seawater. ACS Appl. Mater. Interfaces. 2020, 12, 18012-18022.

(20) Sugo, T.; Tamada, M.; Seguchi, T.; Shimizu, T.; Uotani, M.; Kashima, R. Recovery System for Uranium from Seawater with Fibrous Adsorbent and Its Preliminary Cost Estimation. Journal of the Atomic Energy Society of Japan/Atomic Energy Society of Japan. 2001, 43, 1010-1016.

(21) Tamada, M.; Seko, N.; Kasai, N.; Shimizu, T. Cost Estimation of Uranium Recovery from Seawater with System of Braid Type Adsorbent. Transactions of the Atomic Energy Society of Japan. 2006, 5(4), 358-363.

(22) Tamada, M. Current Status of Technologyc for Collection of Uranium from Seawater (In: International Seminar on Nuclear War and Planetary Emergencies-42nd Session). World Scientific. 2010, $p p, 243-252$.

(23) Baelden, C.; Picard, M.; Wu, Y.; Chang, L.; Slocum, A.-H. Extraction of Uranium from Seawater: Design and Testing of a Symbiotic System. Nucl. Technol. 2013,188 (2), 200-217.

(24) Kim, J.; Tsouris, C.; Oyola, Y.; Janke, C. J.; Mayes, R. T.; Dai, S.; Gill, G.; Kuo, L.-J.; Wood, J.; Choe, K.-Y. Uptake of Uranium from Seawater by Amidoxime-Based Polymeric Adsorbent: Field Experiments, Modeling, and Updated Economic Assessment. Ind. Eng. Chem. Res. 2014, 53(14), 6076-6083. 
(25) Brown, S.; Yue, Y. F.; Kuo, L. J.; Mehio, N.; Li, M. J.; Gill, G.; Tsouris, C.; Mayes, R. T.; Saito, T.; Dai, S. Uranium Adsorbent Fibers Prepared by Atom-Transfer Radical Polymerization (ATRP) from Poly(vinyl chloride)-co-Chlorinated Poly(vinyl chloride) (PVC-co-CPVC) Fiber. Ind. Eng. Chem. Res. 2016, 55(15), 4139-4148.

(26) Oyola, Y.; Janke, C.-J.; Dai, S. Synthesis, Development, and Testing of High-Surface-Area Polymer-Based Adsorbents for the Selective Recovery of Uranium from Seawater. Ind. Eng. Chem. Res. 2016, 55 (15), 4149-4160.

(27) Kuo, L.-J.; Pan, H.-B.; Wai, C. M.; Byers, M. F.; Schneider, E.; Strivens, J. E.; Janke, C. J.; Das, S.; Mayes, R. T.; Wood, J. R.; Schlafer, N.; Gill, G. A. Investigations into the Reusability of Amidoxime-Based Polymeric Adsorbents for Seawater Uranium Extraction. Ind. Eng. Chem. Res. 2017, 56(40), 11603-11611.

(28) Xu, X.; Zhang, H. J.; Ao, J. X.; Xu, L.; Liu, X. Y.; Guo, X. J.; Li, J. Y.; Zhang, L.; Li, Q. N.; Zhao, X. Y.; Ye, B. J.; Wang, D. L.; Shen, F.; Ma, H. J. 3D Hierarchical Porous Amidoxime Fibers Speed Up Uranium Extraction from Seawater. Energy Environ. Sci. 2019, 12 (6), 1979-1988.

(29) Yuan, Y. H.; Zhao, S. L.; Wen, J.; Wang, D.; Gu, X. W.; Xu, L. L.; Wang, X. L.; Wang, N. Rational Design of Porous Nanofiber Adsorbent by Blow-Spinning with Ultrahigh Uranium Recovery Capacity from Seawater. Adv. Funct. Mater. 2019, 29(2), 1805380.

(30) Li, H.; He, N. N.; Cheng, C.; Dong, H.; Wen, J.; Wang, X. L. Antimicrobial Polymer Contained Adsorbent: A Promising Candidate with Remarkable Anti-Biofouling Ability and Durability for Enhanced Uranium Extraction from Seawater. Chem. Eng. J. 2020, 388, 124273.

(31) Li, Z.; Yu, Z.; Wu, Y.; Wu, X.; Wan, Y.; Yuan, Y.; Wang, N. Self-Sterilizing Diblock Polycation-Enhanced Polyamidoxime Shape-Stable Blow-Spun Nanofibers for High-Performance Uranium Capture from Seawater. Chem. Eng. J. 2020, 390, 124648. 
